\title{
Seipin forms a flexible cage at lipid droplet formation sites
}

\author{
Henning Arlt ${ }^{1}{ }^{1,2,3}$, Xuewu Sui ${ }^{1,2}$, Brayden Folger ${ }^{4}$, Carson Adams ${ }^{5}$, Xiao Chen ${ }^{4}$, Roman Remme ${ }^{6}$, \\ Fred A. Hamprecht ${ }^{6}$, Frank DiMaio ${ }^{5}$, Maofu Liao ${ }^{(10}{ }^{2}$, Joel M. Goodman ${ }^{4,8} \bowtie$, Robert V. Farese Jr. ${ }^{1,2,7,8 凶}$ \\ and Tobias C. Walther $1,2,3,7,8 \bowtie$
}

Lipid droplets (LDs) form in the endoplasmic reticulum by phase separation of neutral lipids. This process is facilitated by the seipin protein complex, which consists of a ring of seipin monomers, with a yet unclear function. Here, we report a structure of $S$. cerevisiae seipin based on cryogenic-electron microscopy and structural modeling data. Seipin forms a decameric, cage-like structure with the lumenal domains forming a stable ring at the cage floor and transmembrane segments forming the cage sides and top. The transmembrane segments interact with adjacent monomers in two distinct, alternating conformations. These conformations result from changes in switch regions, located between the lumenal domains and the transmembrane segments, that are required for seipin function. Our data indicate a model for LD formation in which a closed seipin cage enables triacylglycerol phase separation and subsequently switches to an open conformation to allow LD growth and budding.

ipid droplets (LDs) are cellular organelles with a primary function of storing lipids for energy generation and membrane biogenesis ${ }^{1,2}$. They serve as hubs of lipid metabolism, platforms for virus assembly and organizing centers of innate immunity ${ }^{3-5}$. Although cellular LD formation is an evolutionarily conserved, fundamental process, its mechanism is still poorly understood. At its essence, LD biogenesis is the formation of emulsified oil droplets, driven by phase separation of enzymatically synthesized neutral lipids, such as triacylglycerols (TGs), within the lipid bilayer of the endoplasmic reticulum (ER) ${ }^{6,7}$. LDs subsequently bud toward the cytoplasm. LD assembly protein complexes (LDACs) ensure the fidelity of this process and determine where LDs form ${ }^{8,9}$.

A key component of the LDAC is the evolutionarily conserved ER membrane protein seipin. Other LDAC components include several accessory proteins, including LD assembly factor 1 (LDAF1) ${ }^{10}$ in humans or the Ldo proteins in yeast ${ }^{11,12}$. Seipin is encoded by the $B S C L 2$ gene in humans ${ }^{13}$. The importance of seipin in LD formation is emphasized by the phenotypes associated with seipin deficiency. In seipin-deficient yeast cells, LDs form inefficiently with TG blisters accumulating in the $\mathrm{ER}^{14}$. Moreover, LDs in these cells have abnormal protein composition ${ }^{15}$ and unstable junctions with the $\mathrm{ER}^{16}$. Similarly, mammalian cells lacking seipin form many abnormally small LDs with altered protein composition, as well as giant $\mathrm{LDs}^{17}$. In humans, seipin deficiency results in lipodystrophy, multiple organ problems and neurological defects, depending on the mutation?.

Seipin consists of an evolutionarily conserved ER-lumenal domain and flanking transmembrane (TM) segments, and less conserved cytoplasmic N- and C-terminal regions with lengths that vary among species (Extended Data Fig. 1a). Structural analyses show that seipin monomers form a roughy 150 -Å diameter toroid complex, consisting of 12 or 11 subunits in flies or humans, respectively ${ }^{18,19}$. Within the complex, each lumenal domain folds into an $\alpha / \beta$-sandwich domain with resemblance to lipid binding domains ${ }^{18,19}$. This domain is reported to bind negatively charged phospholipids ${ }^{19}$. The lumenal domains form a ring of hydrophobic helices oriented toward the center of the toroid complex and are predicted to insert into the lumenal leaflet of the ER membrane ${ }^{18,20,21}$. In mammals, these helices are necessary for seipin's interaction with LDAF1 (ref. ${ }^{10}$ ), which may be an ortholog of yeast LD organization (Ldo) proteins ${ }^{11,12}$. In contrast to flies or humans, yeast seipin (Sei1) requires another ER protein, Ldb16, for LDAC function in LD biogenesis, which makes yeast an ideal system to dissect separate functions for proteins within LDACs ${ }^{15,22}$. Ldb16 has a long hydrophobic stretch with at least one TM segment, but its function is unclear.

Based on experimental evidence, we proposed that LDACs catalyze neutral lipid accumulation and phase separation of neutral lipids in the ER, generating a neutral lipid lens at the $\mathrm{LDAC}^{10,18}$. Accordingly, LDACs lower the TG concentration at which LD formation occurs in cells ${ }^{10}$. This model is supported by molecular simulation experiments using the lumenal domain structures that detect TG molecules binding and accumulating at seipin's central hydrophobic helices ${ }^{20,21}$. Other models for seipin function include generating or transferring specific lipids to forming $\mathrm{LDs}^{23,24}$, or promoting calcium transport $\mathrm{t}^{25,26}$.

Nonetheless, insights into how seipin and LDACs ensure the fidelity of LD formation have been lacking. One limitation for determining seipin and LDAC function is that structural information and analyses have been restricted so far to seipin's lumenal domain ${ }^{18,19}$. Yet, mutations in this region have relatively minor or variable effects on $\mathrm{LD}$ formation ${ }^{17-19}$, suggesting that crucial determinants of seipin function may lie outside of this domain.

'Department of Molecular Metabolism, Harvard T. H. Chan School of Public Health, Boston, MA, USA. ${ }^{2}$ Department of Cell Biology, Harvard Medical School, Boston, MA, USA. ${ }^{3}$ Howard Hughes Medical Institute, Boston, MA, USA. ${ }^{4}$ Department of Pharmacology, University of Texas Southwestern Medical School, Dallas, TX, USA. ${ }^{5}$ Department of Biochemistry and Institute of Protein Design, University of Washington, Seattle, WA, USA. ${ }^{6}$ Heidelberg Collaborative for Image Processing, Interdisciplinary Center for Scientific Computing, Heidelberg University, Heidelberg, Germany. ${ }^{7}$ Broad Institute of Harvard and MIT, Cambridge, MA, USA. ${ }^{8}$ These authors contributed equally: Joel M. Goodman, Robert V. Farese, Jr., Tobias C. Walther. 凶e-mail: Joel.Goodman@UTSouthwestern.edu; robert@hsph.harvard.edu; twalther@hsph.harvard.edu 
To gain further insight into seipin function, here we combined cryogenic-electron microscopy (cryo-EM) with deep learningguided protein structure prediction based on evolutionary couplings $^{27}$ to generate a near full-length structural model of yeast seipin. Validating and testing these structural predictions provide a new model for how seipin functions in LDACs to catalyze LD formation.

\section{Results}

Seipin's TM segments are crucial for function. We hypothesized that seipin's evolutionarily conserved TM segments are required for LD biogenesis. To test this idea, we replaced either seipin's N-terminal or both TM segments with TM helices from a structurally unrelated, human ER protein, FIT2 (ref. ${ }^{28}$ ). Alternatively, we shuffled the sequences of either $\mathrm{N}$ - or C-terminal TM segments (Extended Data Fig. 1b). The resulting FIT2 chimeras (TM-N-FIT2, TM-C-FIT2, TM-NC-FIT2) and shuffled seipin TM mutants (shuffled-TM-N, shuffled-TM-C, shuffled-TM-NC), which were green fluorescent protein (GFP) tagged, localized in puncta to the ER in a pattern similar to wildtype (WT) seipin (Extended Data Fig. 1c). We also constructed stable lines in which chromosomal seipin was tagged with $13 \mathrm{xMyc}$ and expression driven by either the endogenous promoter or the strong $P G K 1$ promoter, which generally equalized otherwise low expression of mutants (Extended Data Fig. 1d). To test for an effect on oligomer formation, we isolated membranes and examined detergent-solubilized complexes by size-exclusion chromatography. WT seipin-Myc migrated in two peaks: a large complex of an apparent mass well above the $669 \mathrm{kDa}$ marker (below), and a peak at an elution volume corresponding to roughly $300 \mathrm{kDa}$, likely representing micelles containing nonoligomerized seipin. All mutant constructs expressed WT-like oligomers, but each had less of the small $300-\mathrm{kDa}$ peak, likely due to higher turnover of the chimeric or shuffled mutant constructs (Extended Data Fig. 1e). To determine whether the mutant constructs could rescue function, we analyzed the size of LDs in the stable cell lines. WT cells contained multiple small $(r<400 \mathrm{~nm})$, relatively uniform LDs, whereas sei1 $\Delta$ cells typically had tight clusters of small or supersized LDs $\left(r>400 \mathrm{~nm}\right.$; Extended Data Fig. 1f, $\left.{ }^{29,30}\right)$. Neither FIT2, nor shuffled seipin TM segments rescued the null LD phenotype of seipin deletion mutants (Extended Data Fig. 1f-h). Furthermore, none of the mutants fully rescued the growth phenotype of sei1 $\Delta$ cells on media containing terbinafine, a squalene epoxidase inhibitor that serves as an alternative assay for seipin function (Extended Data Fig. 1i, ${ }^{22}$ ). Only the C-terminal, shuffled-TM mutant had some functional activity in this assay. These findings indicate that the TM segments are crucial for seipin function.

Molecular structure of yeast seipin. To better understand seipin function, and particularly the role of its TM segments, we sought to generate a molecular structure for the entire seipin protein. Previous attempts using fly and human seipin proteins did not yield structural information for the TM regions. As an alternative, we purified the yeast seipin Sei1-Ldb16 complex by affinity and size-exclusion chromatography from a strain that overproduced both proteins from the GAL1 promoter at their endogenous loci (Extended Data Fig. 2a,b). After solubilization of membrane fractions in detergent
(Triton X-100), complexes were isolated by affinity chromatography via a $3 x$ FLAG-TEV-2xProteinA tag at the C terminus of Sei1, followed by exchange of detergents to digitonin, cleavage of the $2 x$ Protein A tag with tobacco etch virus (TEV) protease and finally reconstitution of proteins in amphipols (PmalC8). Fractionation by size-exclusion chromatography revealed that the oligomeric Sei1Ldb16 complex migrated at an elution volume corresponding to roughly $600 \mathrm{kDa}$.

Initial processing of negative stain and cryo-EM images of the purified complex yielded a toroid structure of ten subunits (Extended Data Fig. 2c-e). Analysis of this density map with $\mathrm{C}_{10}$ symmetry revealed a region corresponding to seipin ER-lumenal domains resolved to an overall resolution of roughly $3.4 \AA$, but with only weak densities of the TM segments (Extended Data Fig. 2e). The poor resolution of the TM segments might have been due to heterogeneity in the conformations of the TM helices. To explore this possibility, we further classified the cryo-EM particle images without applying symmetry after $\mathrm{C}_{10}$ symmetry expansion (Extended Data Fig. 2e). This revealed that densities from each class of particles visually resembled $\mathrm{C}_{5}$ symmetry. We refined the class with the highest predicted resolution with $\mathrm{C}_{5}$ symmetry, which revealed a roughly 145 - $\AA$ diameter complex with two alternating conformations of the TM segments that were invisible in the three-dimensional (3D) reconstruction with $\mathrm{C}_{10}$ symmetry. We designated these alternate conformations $\mathrm{A}$ and $\mathrm{B}$ (Fig. 1). In this map, nearly all of the lumenal domain of seipin and most of the TM regions are well resolved with an overall resolution of roughly $3.2 \AA$ (Fig. 1a,b and Extended Data Fig. 3a,b).

Based on this EM density map, we built a molecular model of conformation A that included parts of both TM segments and the entire lumenal domain (amino acids (aa) 25-258), except a small segment of residues (aa 134-147) (Extended Data Fig. 3c-e). The EM density for the TM segments of conformation $B$ was of lower resolution than for conformation A (Extended Data Fig. 3a,f), but nevertheless allowed us to manually build an initial model for the lumenal domain and connecting residues to the TM segments (residues 46-234). To build a model for the remainder of both TM segment conformations, we used Rosetta structural modeling, guided by both experimental electron density data and distance and angle constraints generated by a deep neural network (trRosetta) trained to predict contacts from evolutionary couplings (Extended Data Fig. 3g,h, ref. ${ }^{27}$ ). This allowed placement of $\alpha$-helices into the EM densities of conformation A (residues 17-25 and 258-264) and B (residues 17-45 and 235-264), producing a nearly complete model of the seipin protein backbone (Fig. 1c and Extended Data Fig. 3f). This approach also allowed us to extend our model beyond what was resolved in the EM density map that contained almost all of the seipin sequence (conformation A, residues 11-283; conformation B, residues 8-285) (Fig. 1d). Although Ldb16 was detectable in the purified complex (Extended Data Fig. 2b), all the protein density observed by cryo-EM could be unambiguously assigned to seipin.

Our model for yeast seipin revealed a decameric complex with the shape of a domed cage, with the lumenal domains forming the floor of the cage, predicted to sit beneath the lumenal leaflet of the ER membrane (Fig. 1b-d). All lumenal domains of the decameric

Fig. 1 | Cryo-EM structure of yeast seipin Sei1. a, Cryo-EM density map of purified seipin oligomers shows the density of the lumenal domain and TM segments. The five symmetrical subunits are indicated by dashed lines. $\mathbf{b}$, Sideview of cryo-EM density map. Top, overlay of unsharpened density map (semitransparent gray) showing the shape of the micelle, with sharpened map (purple). Bottom, sliced view of EM density map reveals cage-like structure. Position of ER membrane is indicated with gray lines. $\mathbf{c}, \mathbf{d}$, Model of seipin show ten seipin subunits per oligomer. Top view from the cytosolic side. $\mathbf{c}$, Model contains residues 17-264 for both A and B conformations, except loop residues 134-147, which are not observed in the EM density map. d, Extended structural model beyond EM density map contains residues 11-283 for conformation A (blue) and residues 8-285 for conformation B (orange) modeled by $\mathrm{Al}$-assisted structure prediction. e, Seipin oligomers contain two alternating monomer conformations termed A (blue) and B (orange) that differ only in the switch and TM region, while the lumenal domains have the same structure. 
complex had the same structure, with each lumenal domain containing an $\alpha / \beta$-sandwich fold, similar to those in human and fly $\operatorname{seipin}^{18,19}$, and with two short central $\alpha$-helices oriented toward the center ring of the cage floor (Fig. 1c-e). Two 'switch' regions (residues 40-55 and 231-243), representing the biggest differences between conformations $\mathrm{A}$ and $\mathrm{B}$, connect the ring of folded lumenal a

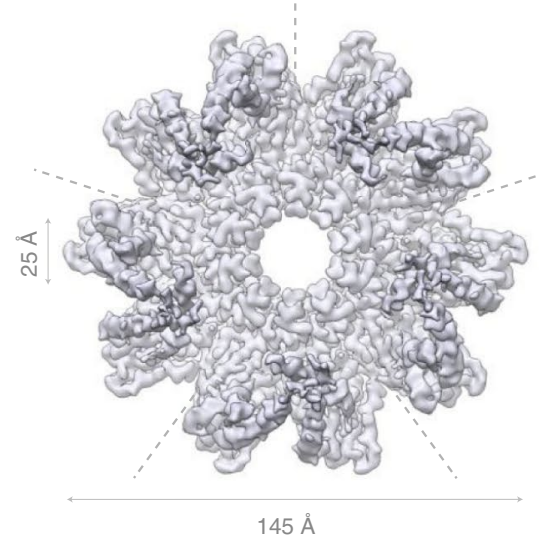

c

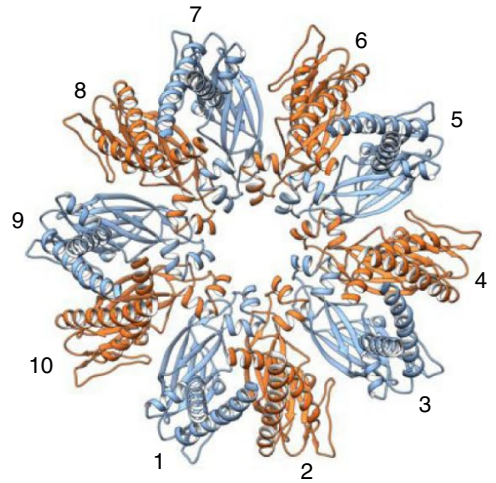

d

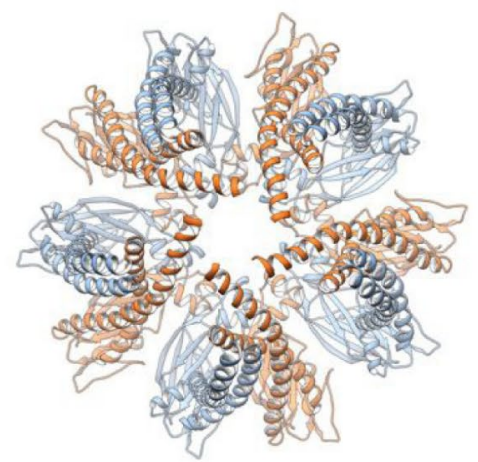

e

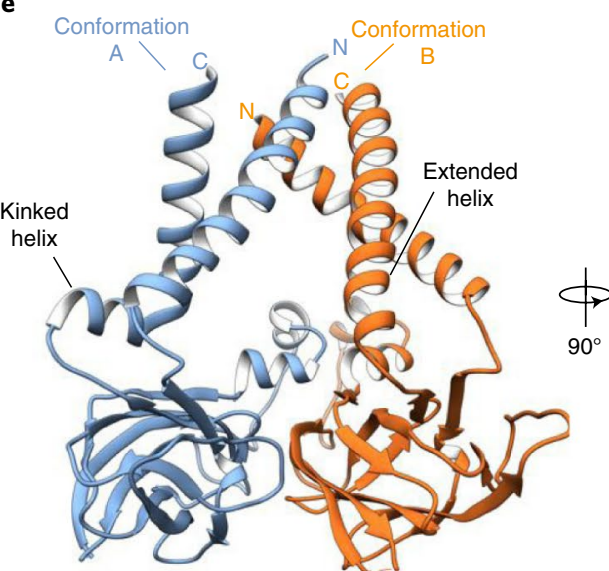

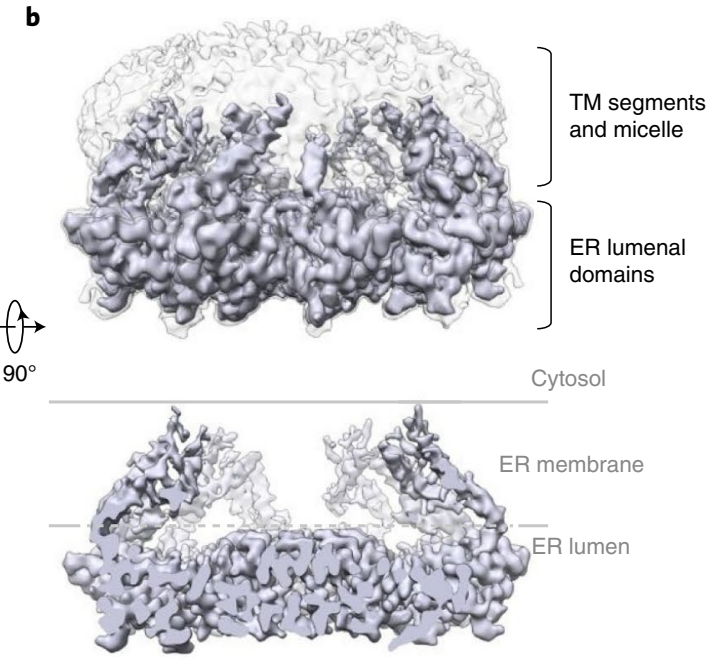
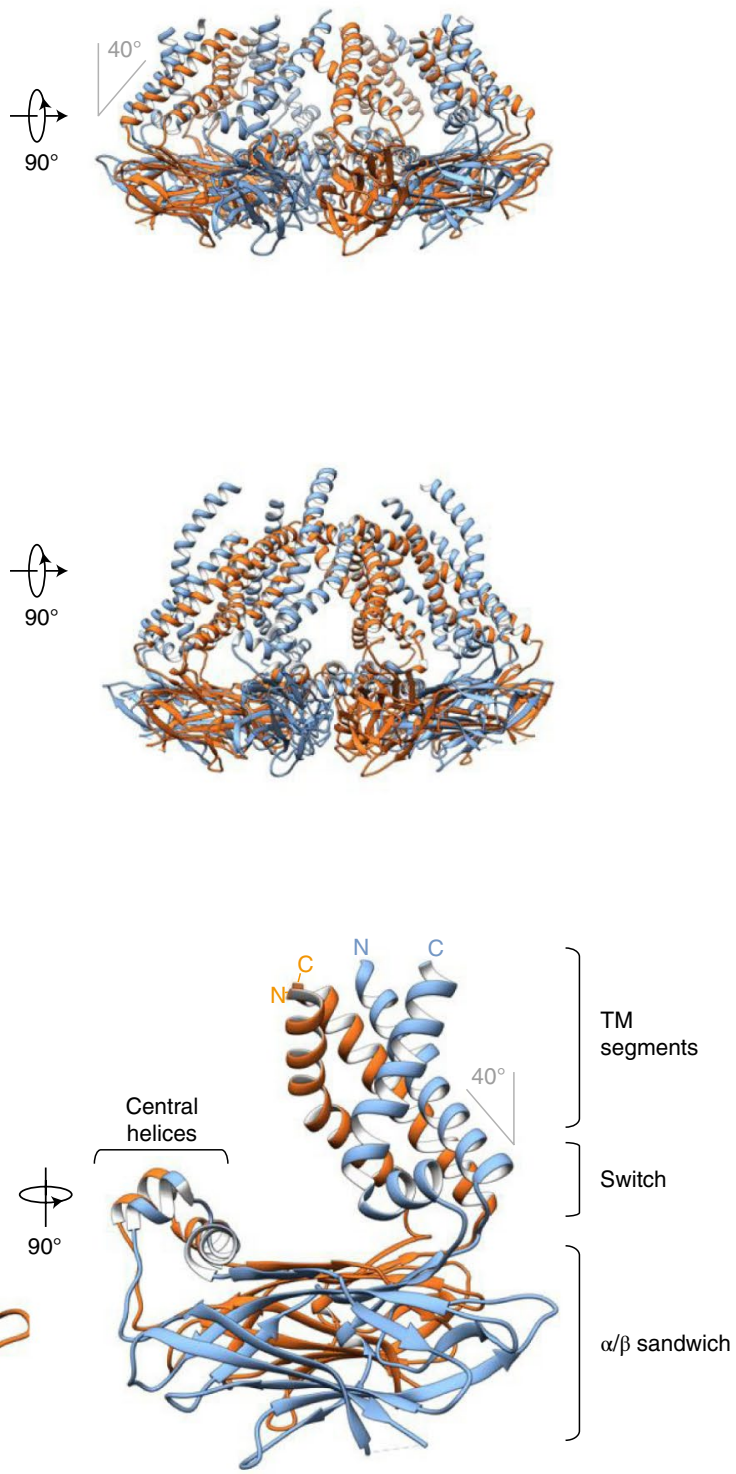
domains to the TM segments of seipin. The TM segments form the side walls of the cage and are tilted toward the center of the oligomer, coming together in a dome-shape at the cytoplasmic side of the complex. The architecture of a cage leads to a large, enclosed cavity in the center of the complex, predicted to be in the plane of the ER membrane (Fig. 1a-c).

In conformation $\mathrm{A}$, the $\mathrm{N}$-terminal TM helix is tilted roughly $40^{\circ}$ toward the center of the oligomer, whereas the C-terminal switch region adopts a kinked $\alpha$-helix connected to the second TM helix (Fig. 1e). In conformation B, the C-terminal TM helix exhibits a continuously extended helix through the switch region and lacks the kink found in conformation A. As a result, the N-terminal TM helix in conformation B is tilted further (roughly $60^{\circ}$ ) toward the center of the oligomeric assembly and both TM segments lie close to the N-terminal TM helix of the neighboring conformation A monomer (Fig. 1c-e).

Lumenal domain interactions are sufficient for oligomerization. Comparing the architecture of individual seipin lumenal domains of yeast with previously determined fly and human protein structures revealed a striking difference (Fig. 2a,b). The fly and human lumenal domains possess a longer central helix that is hydrophobic, interacts with LDAF1 in humans ${ }^{10}$, inserts into the ER bilayer and is implicated in binding TGs in molecular dynamics simulations ${ }^{20,21}$. In contrast, yeast seipin has two short helices with several charged residues (for example, Q169, E172, Q173, E184) and a different orientation compared with human or fly seipin, which likely does not insert into the membrane (Fig. 2a).

To test whether residues in this central lumenal $\alpha$-helix are important for yeast seipin function, we mutated residues Q169, E172 and Q173 or a combination of S167, Q169, E172, Q173, D180, E184 and E185 to alanine $(169,172,173$ to A; $167-1857 x A)$. Alternatively, we deleted the entire helical region and tested the functionality of these mutants in LD formation ( $\Delta 169-173$ or $\Delta 167-174)$. Cells expressing central lumenal $\alpha$-helix mutations did so at normal or moderately reduced levels (Extended Data Fig. 4a) and had LD phenotypes similar to WT (Fig. 2c and Extended Data Fig. 4b,c,). Additionally, each of these mutants complemented growth of seipin-deficient cells on media containing terbinafine (Extended Data Fig. 4d). While sei1s cells had markedly decreased Ldb16 protein levels, mutants of the lumenal $\alpha$-helix had normal or slightly decreased amounts of Ldb16, indicating this region is not required for binding and stabilization of Ldb16 by Sei1 (Extended Data Fig. 4 e) ${ }^{22}$.

Neighboring monomers of the lumenal domains appear to contact each other between residues R178 and E185/W186 of the adjacent monomers (Fig. 2a, inset) to form a hydrogen bond and a salt bridge between R178 and E185 (dotted green lines in Fig. 2a inset) and a cation $-\pi$ interaction between R178 and W186. Because $\mathrm{R} 178$ is central to both interactions, we mutated this residue to alanine to determine whether this interface is required for oligomer formation or stability. C-terminal GFP-tagged seipin R178A localized normally to the ER and formed characteristic GFP-puncta comparable in intensity to the WT protein (Fig. 2d), indicating normal oligomer formation in vivo. We integrated R178A containing a C-terminal $13 x$ myc tag into the endogenous seipin locus, which expressed at reduced levels (Extended Data Fig. 5a), and examined oligomer stability in detergent extracts as described above. Unlike WT seipin that showed two peaks, the R178A mutant showed only the smaller roughly $300-\mathrm{kD}$ peak, and this defect was not corrected by overexpression from the PGK1 promoter (Fig. 2e and Extended Data $5 \mathrm{~b}$ ), suggesting that R178A is important for decamer integrity, at least in detergent-solubilized seipin. A possible hypothesis for Ldb16 function is that it is an assembly factor for seipin complexes. However, deletion of $L D B 16$ had no effect on oligomerization of WT seipin, and overexpression of LDB16 failed to rescue R178A oligomerization (Extended Data Fig. 5b).
Seipin R178A only modestly affected LD morphology (Fig. 2f-h) and fully rescued the terbinafine sensitivity of sei1 $\Delta$ cells (Extended Data Fig. $5 c$ ). Mutation of other residues in the $\alpha / \beta$-sandwich contact region (for example, Q114A and E172A), alone or in combination with R178A, had no effect on LD phenotypes or terbinafine sensitivity in addition to R178A (Extended Data Fig. 5d-f).

We further tested whether seipin lumenal domains are sufficient for decamer formation by expressing a truncated version of the protein lacking TM segments in Escherichia coli $\left(\mathrm{WT}_{47-235}\right.$ ) (Extended Data Fig. 5g-i). By size-exclusion chromatography, the expressed lumenal domain (isolated in the absence of detergent) was sufficient to form oligomers and showed typical ring-shaped decameric assemblies visualized by negative staining EM (Extended Data Fig. 5h,i). Introducing the R178A mutation into the isolated lumenal domains abrogated oligomerization (Extended Data Fig. 5h), indicating that R178 is crucial for assembly of the decamer in the absence of the TM segments and may also be important for stability of the entire protein.

Intramolecular TM interactions are important for function. Molecular dynamics simulations suggest that specific residues of the TM segments of human seipin bind TG to aid in lipid phase separation, which could explain why the TM segments are crucial for seipin function ${ }^{20,21}$. To test this possibility, we mutated three conserved residues in the second TM helix of yeast seipin that were predicted by simulations to interact with TG in human seipin ${ }^{18}$. The mutant yeast protein (Sei1 C260L, S266L, T269I) localized normally to the ER, formed WT-like oligomers, and was able to rescue function analyzed by LD morphology and growth on terbinafine plates (Extended Data Fig. 6a-g), indicating that these residues are not required for yeast seipin function.

These data indicate that other properties of the TM segments are important for its function in LD formation. Within a seipin monomer, both TM segments show close contacts (Fig. 3a). The importance of this is supported by an extensive network of trRosetta-predicted interactions between the N- and C-terminal TM segments (Fig. 3a-c). In particular, two patches of residues coevolved and are predicted to interact within the monomer (for example, residues S33-I259, Y37-Y248, Y41-M240; Fig. 3a-c). To test the requirement for these apparent evolutionary couplings, we mutated specific residues in the N-terminal TM segment (Patch 1, S33A, Y37A, Y41A) or C-terminal TM segment (Patch 2, M240G, Y248I, F255R, I259K). Mutating these patches did not affect seipin localization to the ER, although expression levels were lower than WT, and were restored by inserting the PGK1 promoter (Fig. 3d,e and Extended Data Fig. 7a). Analysis of oligomerization of the patch mutants by size-exclusion chromatography showed WT-like oligomers. However, combinations with the oligomerization mutant R178A led to unfolding or aggregation of the chimeras indicating higher instability of these mutants (Fig. $3 \mathrm{f}$ and Extended Data Fig. 7b), and suggesting that the seipin TM helices normally aid in decamer stability, which becomes critical in the absence of R178 lumenal interactions. Expression of mutants in patch 2, or patches 1 and 2 in sei1 $\Delta$ cells did not maintain seipin function in LD morphology or growth on terbinafine-containing medium, whereas the patch 1 mutant alone rescued the formation of very large LDs and showed intermediate growth on terbinafine plates (Fig. $3 g-i$ and Extended Data Fig. 7c).

Together with previous findings for human seipin ${ }^{10}$, our results highlight the importance of the seipin TM segments for LDAC function. Previously, it was reported that the yeast seipin TM helices are required for interaction with Ldb16 (ref. ${ }^{22}$ ). Western blot analyses of cell lysates expressing the TM helix patch mutants or mutants with exchanged TM segments to FIT2 helices (Extended Data Fig. 1) under control of the PGK1 promoter showed that Ldb16 levels decreased in each of the TM segment mutants to a level generally 

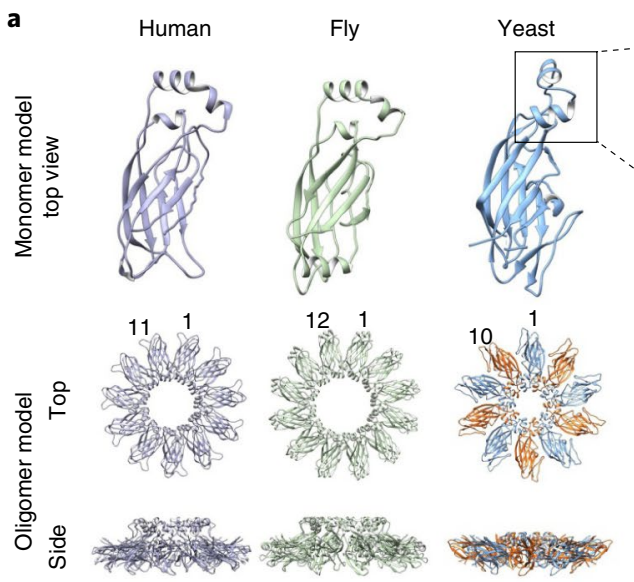

b
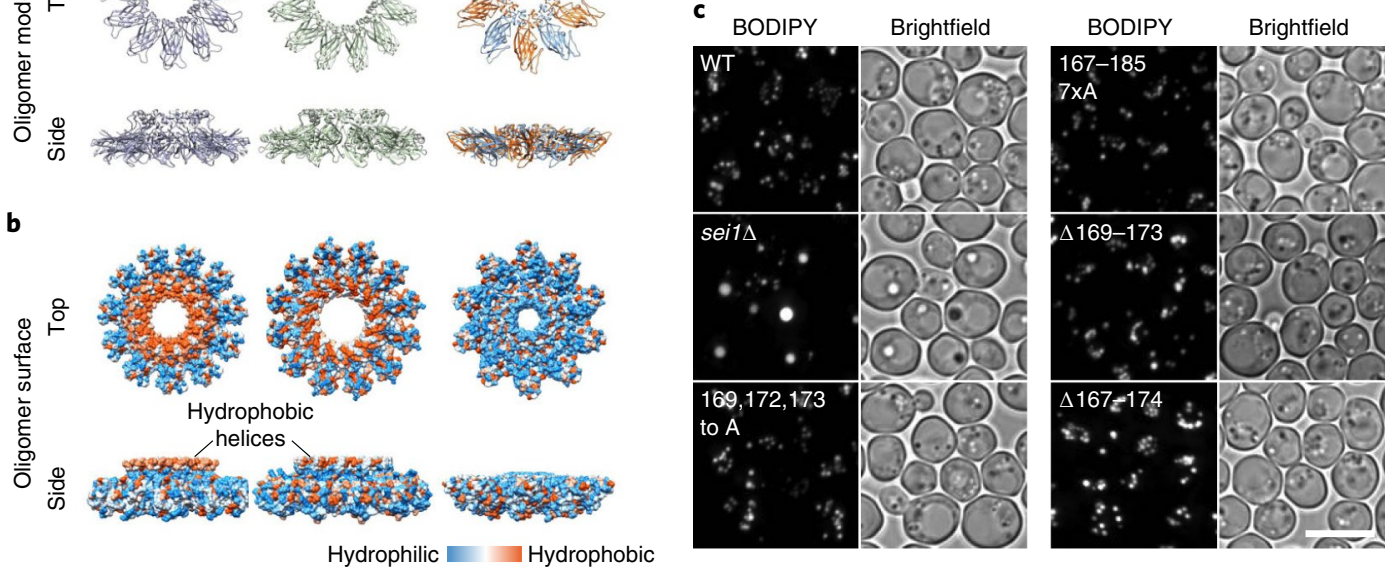

d
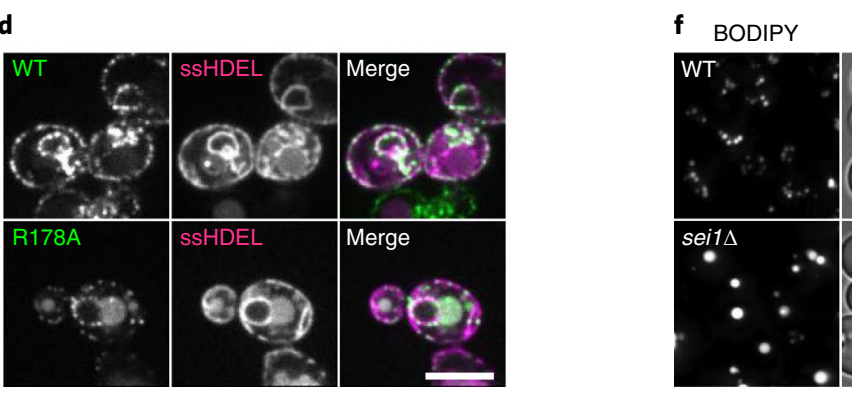

Brightfield

BODIPY
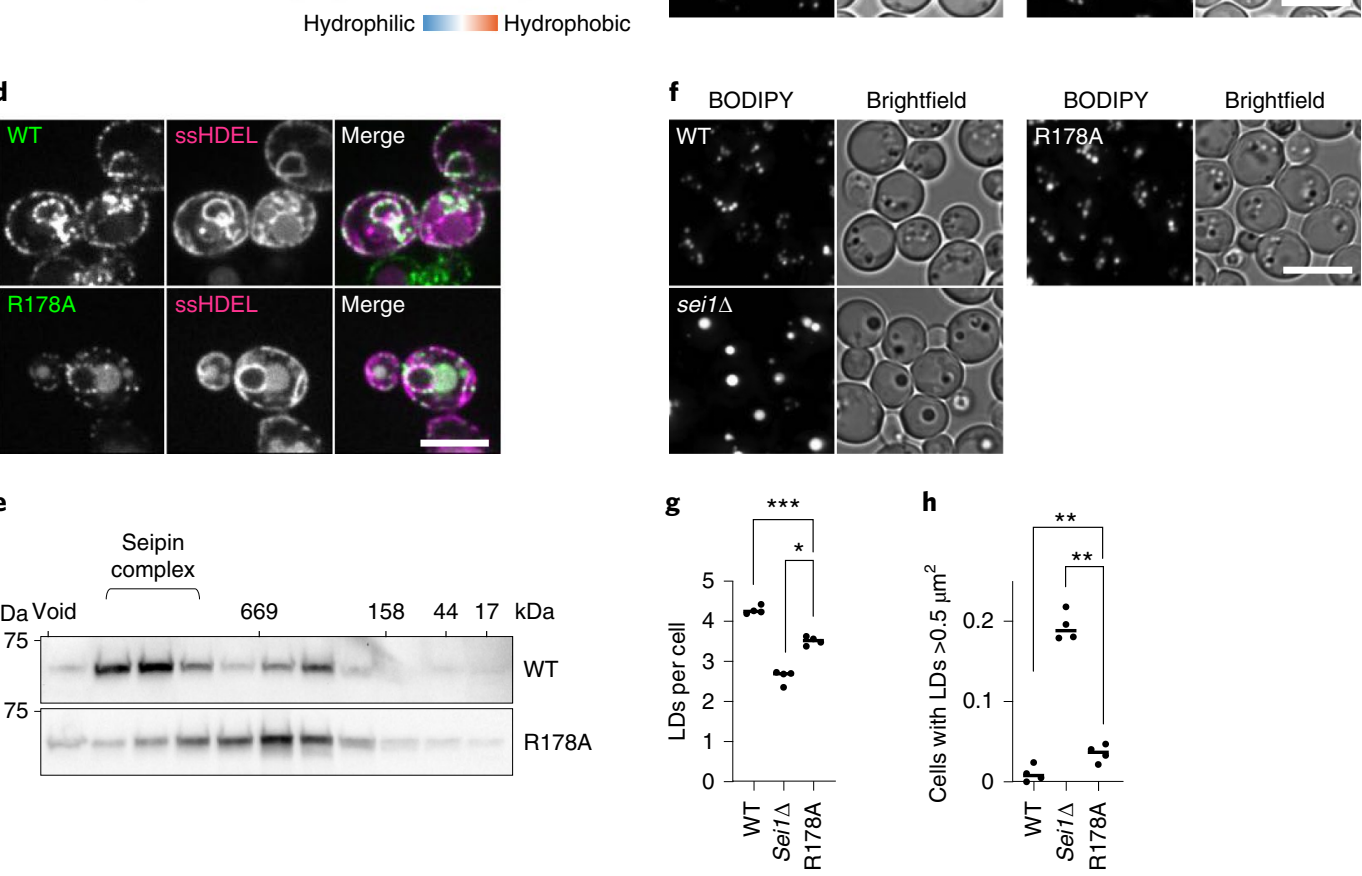

Fig. 2 | Interactions of seipin lumenal domains are sufficient for oligomerization but are not required for seipin function. a, Comparison of seipin lumenal domain structural models of monomers and oligomers from fly (PDB 6MLU), human (PDB 6DS5) and yeast. Magnified box shows detailed view of yeast central helix, including neighboring monomer. b. Hydrophobic surfaces of human, fly and yeast seipin lumenal domains indicate hydrophobic helices present in human and fly, but not yeast seipin. Blue indicates the least hydrophobic and orange the most hydrophobic residues based on the Kyte-Doolittle scale. c, LD morphology of strains expressing central helix mutants from seipin genomic locus. Cells were grown to high density and LDs were stained with BODIPY. Scale bar, $5 \mu \mathrm{m}$. d, WT and R178A localize normally to the ER and form seipin foci. C-terminal GFP-tagged WT and R178A expressed from plasmids in sei1 $\Delta$ cells. ssHDEL was also expressed from a plasmid. Scale bar, $5 \mu \mathrm{m}$. e, Seipin WT shows two peaks in size-exclusion chromatography of membrane extract in Triton X-100 from cell expressing SEI7-13xmyc WT and R178A mutant from endogenous promoter. Immunoblot with anti-myc antibodies. Representative of two biologically independent experiment repeats is shown. $\mathbf{f}$, Microscopy analysis of cell expressing indicated seipin mutants from endogenous locus driven by PGK1 promoter with C-terminal 13xmyc tag or deleted for seipin (sei1 $\Delta$ ). Staining as in c. Scale bar, $5 \mu \mathrm{m} . \mathbf{g}, \mathbf{h}$, Quantification of LD morphology from the experiment shown in $\mathbf{f}$. LDs per cell $(\mathbf{g})$ and cells with LD area $>0.5 \mu m^{2}(\mathbf{h})$ were analyzed from $n=4$ biologically independent experiments. Data were analyzed with one-way ANOVA and Holm-Sidak's post hoc comparisons; ${ }^{\star} P<0.05 ;{ }^{\star \star} P<0.01$; ${ }^{\star * \star} P<0.001$. Graphs indicate mean value; one dot indicates one separate experiment. 


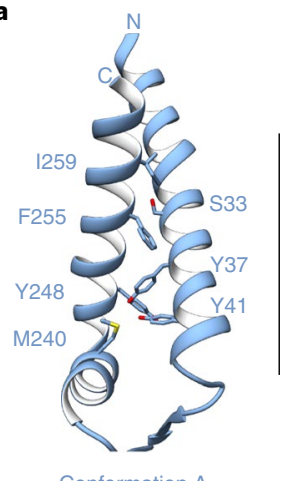

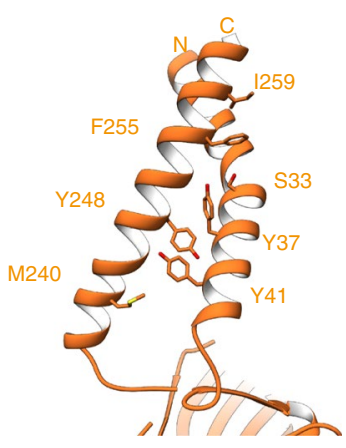

Conformation B

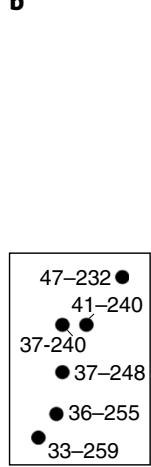

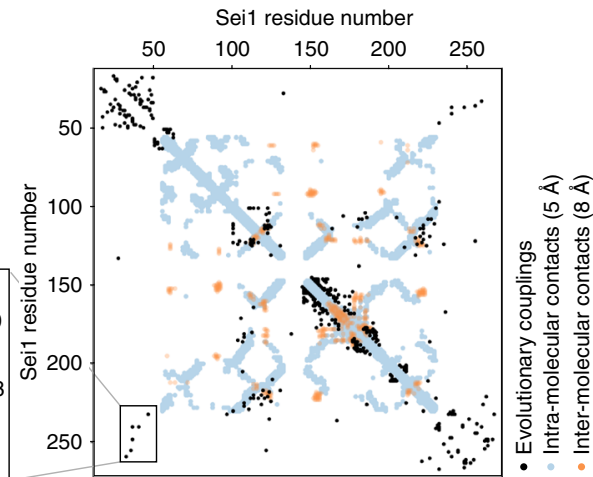

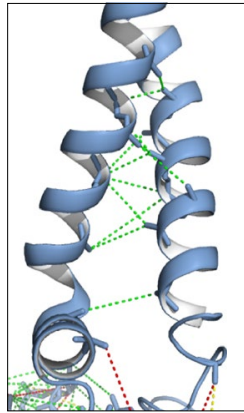

。
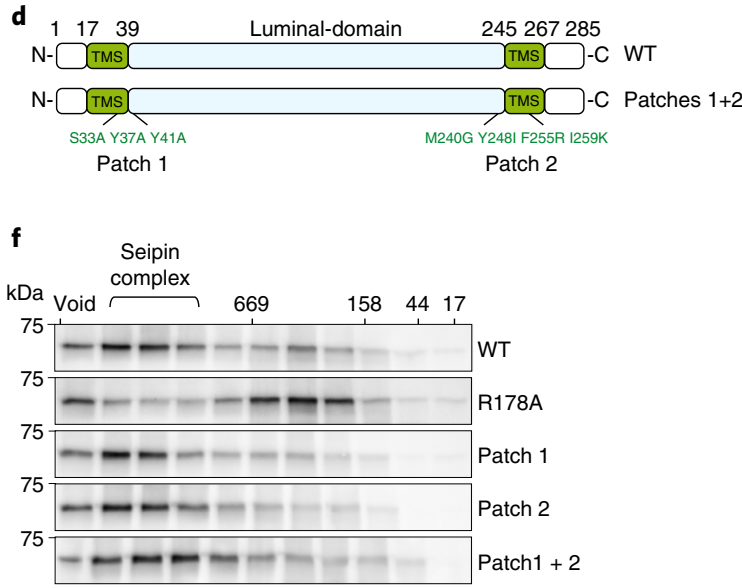

g BODIPY

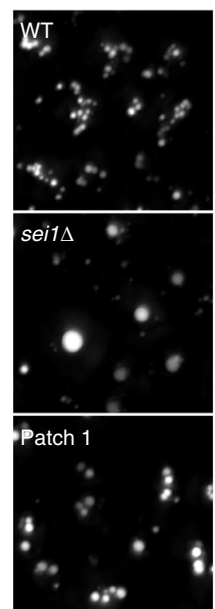

Brightfield

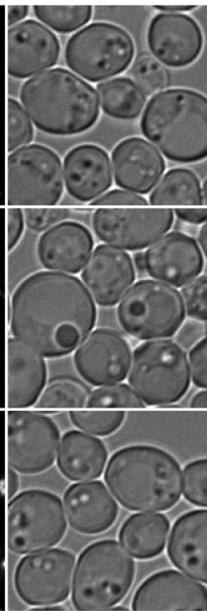

e

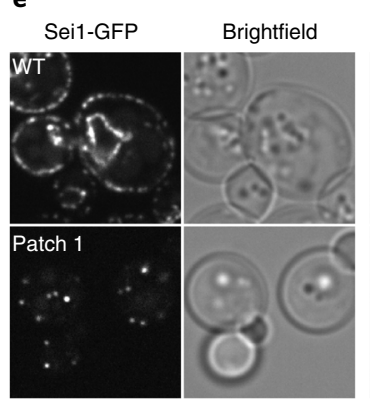

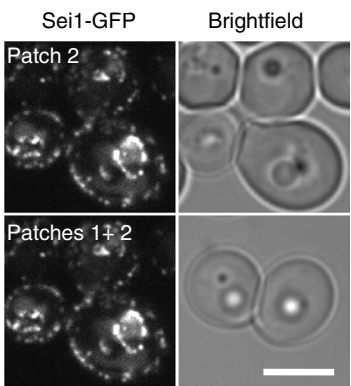

h

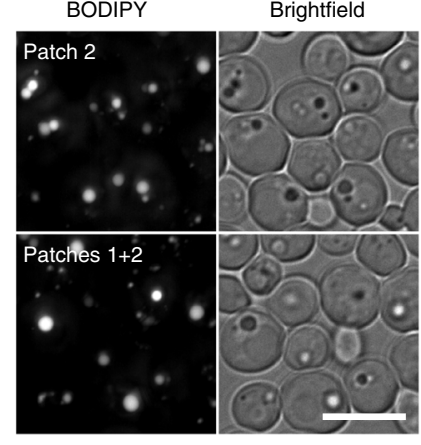

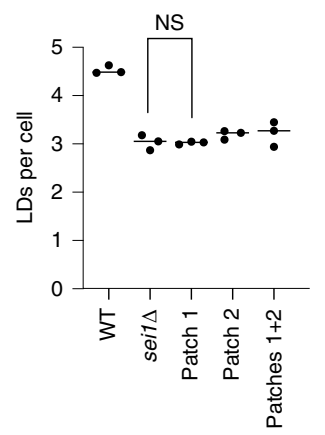

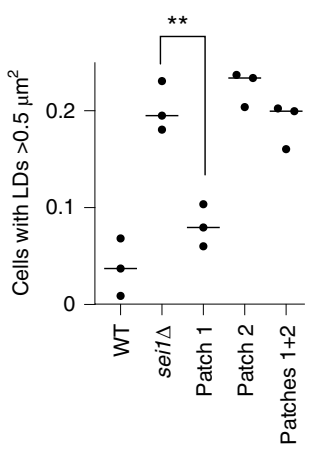

Fig. 3 | TM domain intramolecular interactions are important for seipin function and oligomer formation. a, Detailed view of TM segments and switch regions in conformation $\mathrm{A}$ (blue) and $\mathrm{B}$ (orange); labeled residues are predicted to be involved in intramolecular contacts. $\mathbf{b}$, Evolutionary coupling residues in yeast seipin highlight potential interactions in the TM segment regions. On the left, the membrane-embedded region is magnified. $\mathbf{c}$, Extended seipin structural model of conformation A, showing amino acids at least ten residues apart in the primary sequence predicted to have beta-carbons interacting within $10 \AA$ distance, with maximal probability and over $70 \%$ probability mass, mapped onto the final model. Green dotted lines indicates that the actual distance is within $10 \AA$, yellow within $12 \AA$, and red for $>12 \AA$. View similar to the left side of a.d, Overview of mutant constructs used in this figure. TMS, TM segments. e, Seipin TM segment mutants integrate normally into the membrane and form WT-like foci. Seipin WT and indicated mutants expressed as C-terminal GFP fusion constructs from plasmids in sei1 $\Delta$ cells. Scale bar, $5 \mu \mathrm{m}$. f, Seipin intramolecular TM mutants form normal oligomers. Size-exclusion chromatography of membrane extract in Triton X-100 from cells expressing PGK1 promoter driven seipin and indicated mutants from the endogenous locus with C-terminal 13xmyc tag. Immunoblot with anti-myc antibodies. Representative of two biologically independent experiment repeats is shown. $\mathbf{g}$, LD morphology phenotype of strains expressing patch mutants from PGK1 promoter. Densely grown cells were stained with BODIPY to visualize LDs. Scale bar, $5 \mu \mathrm{m}$. h,i, Quantification of experiment shown in $\mathbf{g}$. One dot equals one separate experiment. LDs per cell (h) and cells containing LDs with area $>0.5 \mu \mathrm{m}^{2}(\mathbf{i})$ analyzed from $n=3$ biologically independent experiments. Data were analyzed with one-way ANOVA and Holm-Sidak's post hoc comparisons; ${ }^{\star \star} P<0.01$; NS, not significant. 
similar to that in sei $1 \Delta$ cells (Extended Data Fig. 7d, ref. ${ }^{22}$ ). However, much of Ldb16 expressed in the TM segment mutants appeared to be able to interact with seipin in pull-down assays (Extended Data Fig. 7e), suggesting that seipin TM segments stabilize Ldb16 but are not strictly necessary for the interaction between the proteins.

Evolutionary coupling predicts intramolecular interactions between TM segments for fly and human seipin, similar to the yeast version of the protein (Extended Data Fig. 8a,b). To test whether the seipin TM helix architecture that we observed in our structure is conserved in evolution, we generated a series of chimeric proteins that contained portions of yeast seipin with regions of either fly or human seipin. Each of the mutants tested rescued yeast seipin deficiency to a similar extent as human or fly seipin (Extended Data Fig. 8c), consistent with previous reports for human $\operatorname{seipin}^{22,29}$. Furthermore, structural predictions of seipin variants from different species by AlphaFold ${ }^{30}$ show an architecture of the TM segments similar to the structure we resolved for conformation A (Extended Data Fig. 8d). In summary, this suggests that the TM architecture is both critical for function and conserved through evolution.

Switch region is required to maintain oligomers and function. The main feature of the two TM segment conformations of the alternating subunits is that the TM helices of conformation B are tilted to the center of the seipin cage and interact with the neighboring TM helices of conformation A (Fig. 1c-e). This architecture is enabled by the flexibility of the switch regions that change most dramatically between conformation $\mathrm{A}$ and $\mathrm{B}$. In particular, the switch region connecting to the seipin C-terminal TM segment showed a marked difference between the $\mathrm{A}$ and $\mathrm{B}$ conformations; it formed a kink in the A conformation but extended into a continuous $\alpha$-helix with the $\mathrm{C}$-terminal TM segment in conformation $\mathrm{B}$ (Figs. 1d and $4 \mathrm{a}$ and Supplementary Video 1). This region also contained a highly conserved $\mathrm{F}_{232} \mathrm{xxGLR}$ sequence motif (Extended Data Figs. 1a and 9a).

To determine whether the switch regions are important for seipin function, we deleted or shuffled their amino acid sequence (residues 46-55 and 231-244; Fig. 4b). The resulting shuffled-switch and $\Delta$-switch mutants showed expression comparable to WT when expressed from the PGK1 promoter (Extended Data Fig. 9b). Disrupting the switch regions dramatically affected the cellular localization of the resulting protein, compared with WT. Instead of seipin foci commonly found at the contact site between the ER and $\mathrm{LDs}^{16,22}$, both mutants formed large rings within the ER that appeared to encircle large LDs, reminiscent of Saturn's rings (Fig. 4c,d). The unusual pattern of switch mutant protein localization prompted us to hypothesize that these mutations weaken the interactions between TM segments of neighboring monomers by changing the arrangements of seipin's A and B conformations. To investigate this possibility, we tested the prediction that complexes of seipin with shuffled-switch regions are less stable in cells.
We found that shuffled and $\Delta$-switch formed smaller oligomers on detergent solubilization as analyzed by size-exclusion chromatography (Fig. 4e).

To test whether the switch regions of seipin are important for function, we assayed the ability of shuffled- and $\Delta$-switch mutants to provide seipin function in vivo. Expression of mutant seipin versions with altered switch regions were unable to complement sei1 $\Delta$ growth on terbinafine and only partially rescued the LD phenotype of sei1 $\Delta$ cells (Fig. $4 \mathrm{f}-\mathrm{i}$ ).

\section{Discussion}

Understanding the function of seipin is crucial to deciphering the mechanism of LD formation from LDACs in the ER. Here we report a structural model for nearly all of the seipin protein of $S$. cerevisiae that combines a high confidence 3.2- $\AA$ molecular model based on cryo-EM of seipin's lumenal domains, the switch regions and TM segments, with an extended molecular model of the TM segments generated by an AI structure-prediction approach.

Core elements of the seipin structure appear to be evolutionarily conserved in yeast, fly and human proteins ${ }^{18,19,31,32}$. The lumenal $\alpha / \beta$-sandwich fold domain is well resolved and has similar features in all species analyzed, except for the centrally located hydrophobic helix. Human and fly seipin have hydrophobic helices protruding into the center of the lumenal ring oligomer, whereas the analogous region in yeast consists of two short helices that are more hydrophilic. In human and fly seipin, the hydrophobic helix region is needed for interaction with LDAF1 (ref. ${ }^{10}$ ) and has been proposed to interact with $\mathrm{TG}^{20,21}$. In yeast, however, we found that mutations of this region had little effect on seipin function. If an analogous central hydrophobic helix is also required in yeast, the yeast-specific Ldb16 protein could provide this function in trans for the LDAC. Because we found no density of Ldb16 in our yeast structure, our study does not address this question. However, a recent report showing crosslinking of Ldb16 to the central helix in yeast provides support for this hypothesis ${ }^{32}$.

The function of the lumenal domain remains uncertain. While this region was reported to bind anionic phospholipids ${ }^{19}$, whether this contributes to seipin function is unknown. Alternatively, the lumenal domain might primarily serve as a structural anchor for forming LDs, positioning key elements of the protein such as the hydrophobic helices at the membrane (for fly and human seipin) and the TM helices at the budding neck. Although the yeast seipin complex contains ten monomers, rather than 12 and 11 subunits in fly and human seipin, respectively ${ }^{18,19}$, the rings formed by the luminal domains of each species are similar in outer diameter and would provide similar diameters to necks of budding LDs.

An important feature of our yeast seipin model is the alternating conformations for monomeric subunit TM segments in the yeast decamer. The regions that change most between the two conformations are the switch regions, which are evolutionarily conserved between species (Extended Data Figs. 1a and 9a). Consistent with

Fig. 4 | The seipin switch regions are required for seipin complex formation and function. a, Detailed view of conformational change in $C$-terminal membrane helix comparing superimposed conformations A and B. Conformation A shows kinked alpha helix, and conformation B has an extended helix. b, Overview of switch mutant constructs. c, Seipin switch mutants forms large ring structures around LDs. Cells expressing C-terminal GFP-tagged seipin and indicated mutants from plasmids in sei1 $\Delta$ cells. LDs were stained with autodot dye. Scale bar, $5 \mu$ m. White box indicated area is shown in $\mathbf{d}$. $\mathbf{d}$, Enlarged view and z-stack of seipin ring structures shown in $\Delta$-switch mutant in c. Scale bar, $1 \mu \mathrm{m}$. e, Shuffled-switch mutant is unable to form WT-like oligomers in detergent extracts. Size-exclusion analysis of membrane extract from cells expressing SEI1-13xmyc or indicated mutants from the endogenous locus driven by integrated PGK1 promoter. Representative immunoblots of two biologically independent experiment repeats is shown. $\mathbf{f}$, Growth of yeast strain sei1 $\Delta$ carrying plasmids with C-terminally GFP-tagged SEII (WT), or indicated mutants on synthetic medium $\pm 100 \mu \mathrm{m} \mathrm{ml}^{-1}$ terbinafine. $\mathbf{g}$, LD morphology analysis of strains shown in e. Scale bar, $5 \mu \mathrm{m}$. h,i, Quantification of LD morphology analysis shown in $\mathbf{f}$. LDs per cell (h) and cells containing LDs with area $>0.5 \mu m^{2}$ (i) from $n=3$ biologically independent experiments. Data were analyzed with one-way ANOVA and Holm-Sidak's post hoc comparisons; ${ }^{\star} P<0.05$; NS, not significant. $\mathbf{j}$, Model of seipin function in TG phase separation and LD budding by changing conformations of the TM segments. Left side shows the conformation we obtained experimentally, and right side a predicted version of an 'open' conformation based on all TM segments in the A conformation. Bottom model shows side views with TG accumulation in the complex. 
our findings, ab initio structure prediction using the AI-system AlphaFold predicts that the TM segments of various metazoan seipins have a conformation similar to our experimentally determined structure of yeast seipin conformation A (Extended Data Fig. 8d, ref. ${ }^{33}$ ). Inasmuch as salient features of protein machines are most often conserved evolutionarily, we consider it likely that similar alternative conformations for the TM segments are possible for the human, worm or fly proteins. However, although fly seipin with 12 monomers could adopt a symmetrical arrangement of A/B conformations, such symmetrical alternating conformations would be impossible for the 11-mer reported for human seipin ${ }^{19}$. This suggests that either human seipin complexes may be asymmetric, or that seipin can contain a mix of A and B conformations at any given time in vivo, such that symmetry in this respect is not important.

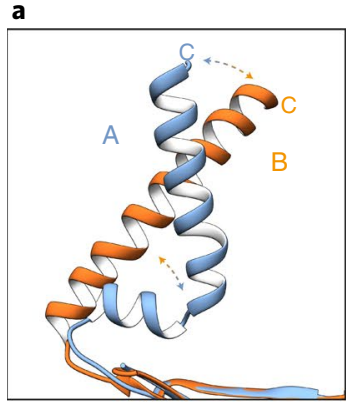

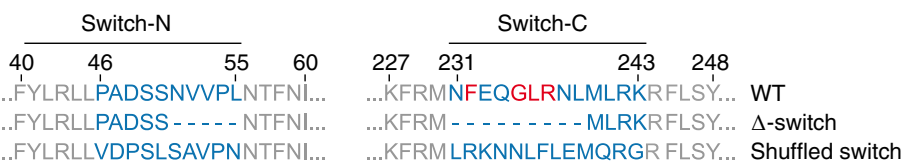
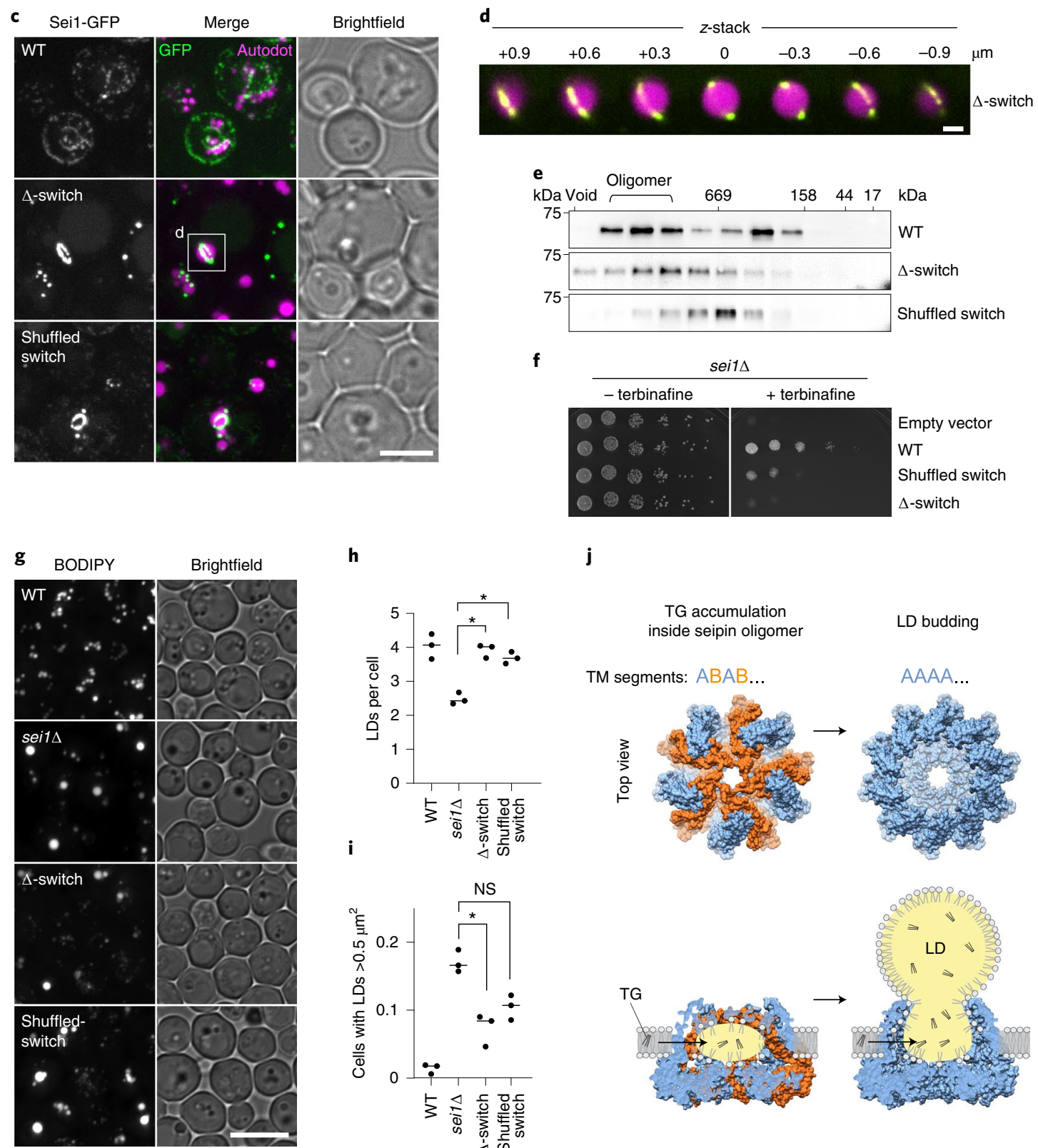

h

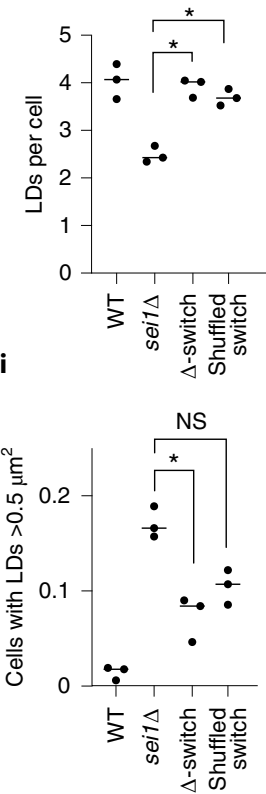

j

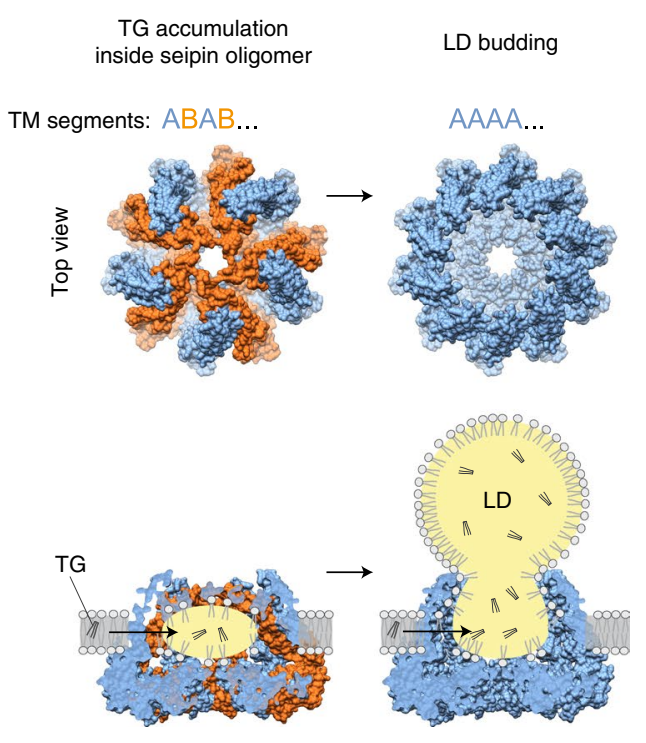


Considering our findings and data available from previous reports, we propose a molecular model for seipin function during LD biogenesis. In this model, the seipin cage sits in the ER and provides a space where its many TM segments (and those of other LDAC proteins) serve to generate a space in the bilayer that is relatively poor in phospholipids. We hypothesize that this space in LDACs allows for TG molecules to interact with each other, rather than with phospholipid acyl chains. TGs, and possibly other neutral lipids, may diffuse into the complex through gaps in the plane of the membrane between seipin monomers. The TM segments may aid this process by binding of TG as proposed by molecular simulations ${ }^{20,21}$, but we did not find evidence for this mechanism in our studies (Extended Data Fig. 6). In any event, the net result is that seipin would allow interactions of TG molecules, thus catalyzing TG phase separation, lens formation and growth. As the TG lens grows, the seipin oligomer may open toward the cytoplasm, with all subunits in the A conformation, and thus release the lens to generate an LD bud (Fig. 4j). As the forming LD grows, the TM segments could further tilt away from the center of the dome to accommodate the growing LDs. In agreement with this interpretation, our cryo-EM data indicate a higher degree of flexibility in the TM segments toward the cytoplasmic side of the seipin complex.

To maintain the neck of ER with LDs, and to allow this change in architecture, the switch region and interactions of TM segments would be particularly important. Consistent with this model, mutants in the switch region appear to lead to a seipin complex that cannot maintain a constricted neck at the ER-LD junction but rather dissociates and integrates more seipin subunits, eventually forming the large-diameter ring structures that we found around large LDs (Fig. 4c,d). Possibly related to such interactions, larger diameter rings of seipin form around LDs in C. elegans ${ }^{34}$.

Our model provides a conceptual framework for seipin function that can now be further tested by experiments and molecular modeling. It will be important to also integrate the structures and functions of additional known LDAC components, such as Ldb16 and the Ldo proteins in yeast, or LDAF1 in humans. Testing and refinement of the model should result in an increasingly clear understanding of this elegant protein machinery that governs the process of making oil droplets in cells.

\section{Online content}

Any methods, additional references, Nature Research reporting summaries, source data, extended data, supplementary information, acknowledgements, peer review information; details of author contributions and competing interests; and statements of data and code availability are available at https://doi.org/10.1038/ s41594-021-00718-y.

Received: 5 August 2021; Accepted: 21 December 2021; Published online: 24 February 2022

\section{References}

1. Walther, T. C. \& Farese, R. V. Jr. Lipid droplets and cellular lipid metabolism. Annu. Rev. Biochem. 81, 687-714 (2012).

2. Zechner, R., Madeo, F. \& Kratky, D. Cytosolic lipolysis and lipophagy: two sides of the same coin. Nat. Rev. Mol. Cell Biol. 18, 671-684 (2017).

3. Monson, E. A. et al. Intracellular lipid droplet accumulation occurs early following viral infection and is required for an efficient interferon response. Nat. Commun. 12, 4303 (2021).

4. Masaki, T. et al. Interaction of hepatitis $\mathrm{C}$ virus nonstructural protein $5 \mathrm{~A}$ with core protein is critical for the production of infectious virus particles. J. Virol. 82, 7964-7976 (2008).

5. Graef, M. Lipid droplet-mediated lipid and protein homeostasis in budding yeast. FEBS Lett. 592, 1291-1303 (2018).

6. Khandelia, H., Duelund, L., Pakkanen, K. I. \& Ipsen, J. H. Triglyceride blister in lipid bilayers: implications for lipid droplet biogenesis and the mobile lipid signal in cancer cell membranes. PLoS ONE 5, e12811 (2010).

7. Thiam, A. R. \& Ikonen, E. Lipid droplet nucleation. Trends Cell Biol. 31, 108-118 (2021).
8. Choudhary, V., El Atab, O., Mizzon, G., Prinz, W. A. \& Schneiter, R. Seipin and Neml establish discrete ER subdomains to initiate yeast lipid droplet biogenesis. J. Cell Biol. 219, e201910177 (2020).

9. Rao, M. J. \& Goodman, J. M. Seipin: harvesting fat and keeping adipocytes healthy. Trends Cell Biol. 31, 912-923 (2021).

10. Chung, J. et al. LDAF1 and seipin form a lipid droplet assembly complex. Dev. Cell 51, 551-563.e7 (2019).

11. Eisenberg-Bord, M. et al. Identification of seipin-linked factors that act as determinants of a lipid droplet subpopulation. J. Cell Biol. 217, 269-282 (2018).

12. Teixeira, V. et al. Regulation of lipid droplets by metabolically controlled Ldo isoforms. J. Cell Biol. 217, 127-138 (2018).

13. Magré, J. et al. Identification of the gene altered in Berardinelli-Seip congenital lipodystrophy on chromosome 11q13. Nat. Genet. 28, 365-370 (2001).

14. Cartwright, B. R. et al. Seipin performs dissectible functions in promoting lipid droplet biogenesis and regulating droplet morphology. Mol. Biol. Cell 26, 726-739 (2015)

15. Grippa, A. et al. The seipin complex Fld1/Ldb16 stabilizes ER-lipid droplet contact sites. J. Cell Biol. 211, 829-844 (2015).

16. Salo, V. T. et al. Seipin regulates ER-lipid droplet contacts and cargo delivery. EMBO J. 35, 2699-2716 (2016).

17. Wang, H. et al. Seipin is required for converting nascent to mature lipid droplets. eLife 5, e16582 (2016).

18. Sui, X. et al. Cryo-electron microscopy structure of the lipid droplet-formation protein seipin. J. Cell Biol. 217, 4080-4091 (2018).

19. Yan, R. et al. Human SEIPIN binds anionic phospholipids. Dev. Cell 47, 248-256.e4 (2018).

20. Prasanna, X. et al. Seipin traps triacylglycerols to facilitate their nanoscale clustering in the endoplasmic reticulum membrane. PLoS Biol. 19, e3000998 (2021).

21. Zoni, V. et al. Seipin accumulates and traps diacylglycerols and triglycerides in its ring-like structure. Proc. Natl Acad. Sci. USA 118, e2017205118 (2021).

22. Wang, C. W., Miao, Y. H. \& Chang, Y. S. Control of lipid droplet size in budding yeast requires the collaboration between Fld1 and Ldb16. J. Cell Sci. 127, 1214-1228 (2014).

23. Pagac, M. et al. SEIPIN regulates lipid droplet expansion and adipocyte development by modulating the activity of glycerol-3-phosphate acyltransferase. Cell Rep. 17, 1546-1559 (2016).

24. Sim, M. F. M. et al. Oligomers of the lipodystrophy protein seipin may co-ordinate GPAT3 and AGPAT2 enzymes to facilitate adipocyte differentiation. Sci. Rep. 10, 3259 (2020).

25. Bi, J. et al. Seipin promotes adipose tissue fat storage through the ER $\mathrm{Ca}^{2+}$-ATPase SERCA. Cell Metab. 19, 861-871 (2014)

26. Ding, L. et al. Seipin regulates lipid homeostasis by ensuring calcium-dependent mitochondrial metabolism. EMBO J. 37, e97572 (2018).

27. Yang, J. et al. Improved protein structure prediction using predicted interresidue orientations. Proc. Natl Acad. Sci. USA 117, 1496-1503 (2020).

28. Becuwe, M. et al. FIT2 is an acyl-coenzyme A diphosphatase crucial for endoplasmic reticulum homeostasis. J. Cell Biol. 219, e202006111 (2020).

29. Fei, W. et al. Fld1p, a functional homologue of human seipin, regulates the size of lipid droplets in yeast. J. Cell Biol. 180, 473-482 (2008).

30. Senior, A. W. et al. Improved protein structure prediction using potentials from deep learning. Nature 577, 706-710 (2020).

31. Szymanski, K. M. et al. The lipodystrophy protein seipin is found at endoplasmic reticulum lipid droplet junctions and is important for droplet morphology. Proc. Natl Acad. Sci. USA 104, 20890-20895 (2007).

32. Klug, Y. A. et al. Mechanism of lipid droplet formation by the yeast Sei1/Ldb16 Seipin complex. Nat. Commun. 12, 5892 (2021).

33. Jumper, J. et al. Highly accurate protein structure prediction with AlphaFold. Nature 596, 583-589 (2021).

34. Cao, Z. et al. Dietary fatty acids promote lipid droplet diversity through seipin enrichment in an ER subdomain. Nat. Commun. 10 $2902(2019)$.

Publisher's note Springer Nature remains neutral with regard to jurisdictional claims in published maps and institutional affiliations.

Open Access This article is licensed under a Creative Commons Attribution 4.0 International License, which permits use, sharing, adaptation, distribution and reproduction in any medium or format, as long as you give appropriate credit to the original author(s) and the source, provide a link to the Creative Commons license, and indicate if changes were made. The images or other third party material in this article are included in the article's Creative Commons license, unless indicated otherwise in a credit line to the material. If material is not included in the article's Creative Commons license and your intended use is not permitted by statutory regulation or exceeds the permitted use, you will need to obtain permission directly from the copyright holder. To view a copy of this license, visit http://creativecommons.org/licenses/by/4.0/.

(c) The Author(s) 2022 


\section{Methods}

Plasmids. All plasmids used in this study are listed in Supplementary Table 1. A yeast shuttle vector, $\mathrm{pBMF} 1$, a derivative of $\mathrm{pRS} 313$, was constructed that contained the following in tandem, flanked by HindIII and SacII sites: 207 basepair (bp) SEI1 5' untranslated region, SEI1 coding region in frame with 13 copies of sequence encoding the myc epitope, ADH1 terminator, the pRS313 HIS3 cassette and 236 bp of SEI1 $3^{\prime}$ untranslated region. SEI1 mutants were generated in PBMF1 using appropriate PCR products and NEBuilder HiFi DNA Assembly (New England Biolabs).

A plasmid, $\mathrm{pSK}^{-}$-NAT-PGK, was constructed containing the nourseothricin resistance cassette (from pFA6-natMX6) and the PGK1 promoter (982 bp of 5' untranslated sequence). For overexpression of seipin mutants, a PCR product containing the NAT-PGK1 fragment was inserted upstream of the seipin coding region in the genome.

For expression of C-terminal GFP-tagged constructs, SEII or gene-synthesized mutants (Integrated DNA Technologies) flanked by HindIII and BamHI were inserted into a pRS416 vector containing $A D H 1$ promoter (705bp) and GFP. pHA234 expressing GFP alone served as empty vector control.

All PCR-derived fragments were fully sequenced in plasmids and mutations in the genome verified.

Yeast strains. All strains (Supplementary Table 2) were based on a W303-1 $\mathrm{A}^{35}$ or BY4741 background. PLN1 was knocked out when indicated by replacement with a hygromycin-resistance cassette. Seipin mutants were generated by transforming yeast with a the HindIII-SacII DNA fragment from pBMF1 (with appropriate mutations) containing SEI1 sequences and the HIS3 marker for selection of transformed clones. Homologous recombination was confirmed by PCR and mutations in the genome confirmed by sequencing.

Insertion of GAL1 promoters and C-terminal 3xFLAG-TEV-2xProteinA tag to generate HAY60 was carried out by integration of PCR products from plasmids pYM-N22, pYM-N23 (ref. ${ }^{36}$ ) and pFA6a-hphMX-(3×FLAG)-TEV-ProtA (gift from M.N. Boddy, Addgene plasmid no. 52692).

For antibiotic selection, strains were selected on yeast peptone $2 \%$ dextrose (YPD) plates containing nourseothricin (GoldBio), hygromycin B (Thermo Fisher Scientific) or kanamycin (Sigma-Aldrich). Transformants were first grown overnight on YPD plates before stamping onto antibiotic plates or directly plated onto antibiotic plates after incubation in YPD shaking culture for $3 \mathrm{~h}$ at $30^{\circ} \mathrm{C}$.

Protein expression and purification. Sei1-Ldb16 complexes were expressed from yeast strain HAY60 grown in yeast peptone media supplemented with $2 \%$ galactose (YPG) for at least $24 \mathrm{~h}$ at $30^{\circ} \mathrm{C}$ in 1-1 cultures. Densely grown cells were collected by centrifugation $(15,900 \mathrm{~g})$, and were washed once with water and buffer A (50 mM Tris pH 8.0, $150 \mathrm{mM} \mathrm{NaCl}, 0.5 \mathrm{mM}$ EDTA, $10 \%$ glycerol). Cell pellets were resuspended in a small volume of buffer A supplemented with $35 \mu \mathrm{l} \mathrm{ml}^{-1}$ yeast protease inhibitor cocktail (Sigma) and snap frozen in liquid nitrogen. Frozen cell pellets were lysed in a cryo-mill, and ground lysate powder was stored at $-80^{\circ} \mathrm{C}$. For large purifications, typically $100 \mathrm{~g}$ of powder from roughly $10-1$ cultures was thawed at room temperature, supplemented with buffer $\mathrm{A}$, and followed by centrifugation at $4,000 \mathrm{~g}$ for $10 \mathrm{~min}$ to remove cell debris. Membranes were isolated by ultracentrifugation at $125,000 \mathrm{~g}$ for $1 \mathrm{~h}$ at $4^{\circ} \mathrm{C}$, were resuspended in buffer A containing $1 \%$ Triton X-100 for $1-2 \mathrm{~h}$ at $4^{\circ} \mathrm{C}$ and centrifuged again for $1 \mathrm{~h}$ at $125,000 \mathrm{~g}$. The supernatant was incubated for $2 \mathrm{~h}$ with $6 \mathrm{ml}$ of washed $\operatorname{IgG}$ Sepharose 6 Fast Flow beads (Cytiva) at $4^{\circ} \mathrm{C}$ on a nutator. Beads were washed with $10 \mathrm{ml}$ buffer B ( $50 \mathrm{mM}$ Tris pH 8.0, $150 \mathrm{mM} \mathrm{NaCl}, 5 \mathrm{mM} \mathrm{MgCl}_{2}$ ) $+0.05 \%$ Triton $\mathrm{X}$-100, twice in same buffer $+0.5 \mathrm{mM}$ ATP, $2 \times 6 \mathrm{ml}$ of buffer B without detergent and $6 \mathrm{ml}$ of buffer B with $0.1 \%$ digitonin. Sei1-Ldb16 complexes were eluted by TEV-cleavage using home-made TEV protease in $3 \mathrm{ml}$ of buffer $\mathrm{B}+0.1 \%$ digitonin overnight at $8^{\circ} \mathrm{C}$ with constant shaking ( 350 r.p.m.). The eluate was concentrated in $100-\mathrm{kDa}$ filters (Amicon) and separated on a Superose 6 Inc column in buffer $\mathrm{B}+0.05 \%$ digitonin. Protein-containing fractions were combined and concentrated to $1.5-\mathrm{ml}$ volume, and 1:3 (w/w) PmalC8 (Anatrace) was added. Mixture was loaded to $35-\mathrm{kDa}$ dialysis filters in $50-\mathrm{ml}$ falcons to buffer $\mathrm{B}$, supplemented with $500 \mu \mathrm{l}$ of Bio-Beads SM-2 (Bio-Rad) overnight at $4^{\circ} \mathrm{C}$ on a nutator. PmalC8-reconstituted protein complexes were subjected to another size-exclusion chromatography on Superose 6 Increase column in buffer B (Extended Data Fig. 2a) and used for negative staining or cryo-EM sample preparation and western blot analysis using anti-FLAG (Sigma; diluted 1:5,000) and anti-Ldb16 antibodies $(22$, diluted 1:3,000)

WT and R178A seipin lumenal domains were expressed in SHuffle T7 Express E. coli cells using plasmids pHA147 $\left(\mathrm{WT}_{(47-235)}\right)$ and $\mathrm{pHA} 144\left(\mathrm{R}_{\left.178 \mathrm{~A}_{(47-235)}\right)}\right)$ that contained a C-terminal 6xHis tag. After induction at an optical densiry $\left(\mathrm{OD}_{600}\right)$ of 0.8 with $0.5 \mathrm{mM}$ isopropylthio- $\beta$-galactoside and incubation at $16^{\circ} \mathrm{C}$ overnight, cells were gathered and lysed in buffer C $(50 \mathrm{mM}$ Tris pH $8.0,400 \mathrm{mM} \mathrm{NaCl}$, $5 \mathrm{mM} \mathrm{MgCl}_{2}$ ) supplemented with $1 \mathrm{mM}$ phenylmethylsulfonyl fluoride and $20 \mathrm{mM}$ imidazole in a Microfluidizer LM 20 (Microfluidics) run at 18,000 PSI. Lysate was cleared by centrifugation for $30 \mathrm{~min}$ at $20,000 \mathrm{~g}$ at $4^{\circ} \mathrm{C}$, and supernatant was incubated with Ni-NTA agarose beads for $1 \mathrm{~h}$ at $4^{\circ} \mathrm{C}$. Beads were collected and washed with buffer $\mathrm{C}+5 \%$ glycerol and $40 \mathrm{mM}$ imidazole, followed by elution in buffer $\mathrm{C}+5 \%$ glycerol and $500 \mathrm{mM}$ imidazole. Purified proteins were analyzed by size-exclusion chromatography using Superdex 200 Increase column in buffer C $+5 \%$ glycerol.
Table 1 | Cryo-EM data collection, refinement and validation statistics

Yeast seipin oligome (EMDB-24674) (PDB 7RSL)

\section{Data collection and processing}

Magnification

105,000

Voltage (kV)

300

Electron exposure $\left(\mathrm{e}^{-} / \AA^{2}\right)$

28.73

Defocus range $(\mu \mathrm{m})$

$-1.2,-2.5$

Pixel size ( $(\AA)$

0.825

Symmetry imposed

Initial particle images (no.)

C5

Final particle images (no.)

1.1 million

Map resolution ( $\AA$ )

49,028

FSC threshold

Map resolution range $(\AA)$

\section{Refinement}

Initial model used (PDB code)

Model resolution $(\AA)$

3.45

FSC threshold

0.5

Model resolution range $(\AA)$

Map sharpening $B$ factor $\left(\AA^{2}\right)$

$-75$

Model composition

Nonhydrogen atoms

19,150

Protein residues

2,350

Ligands

0

$B$ factors $\left(\AA^{2}\right)$

Protein

Ligand

NA

R.m.s. deviations

Bond lengths $(\AA)$

Bond angles $\left({ }^{\circ}\right)$

\section{Validation}

MolProbity score $\quad 1.87$

Clashscore $\quad 11.08$

Poor rotamers (\%) $\quad 0.23$

Ramachandran plot

Favored (\%)

Allowed (\%)

4.50

Disallowed (\%)

0

Size-exclusion analysis of membrane extracts. Yeast strains expressing SEI1$13 x m y c$ were grown in $25 \mathrm{ml}$ of YPD culture at $30^{\circ} \mathrm{C}$ for $16-24 \mathrm{~h}$, and were harvested and washed with water by centrifugation at $4,000 \mathrm{~g}$ for $5 \mathrm{~min}$. Cell pellets were resuspended in $600 \mu \mathrm{l}$ of buffer A with protease inhibitor cocktail. Next, $250 \mu \mathrm{l}$ of $0.5-\mathrm{mm}$ silica beads were added, and cells lysed in a bead beater $2 \times 30 \mathrm{~s}$ at full speed with 10-min breaks on ice. Lysate was harvested by centrifugation $(20 \mathrm{~s}, 18,000 \mathrm{~g})$ and precleared $\left(5,000 \mathrm{~g}, 10 \mathrm{~min}, 4^{\circ} \mathrm{C}\right)$. Membranes were collected by centrifugation at $125,000 \mathrm{~g}$ for $1 \mathrm{~h}$ and solubilized in buffer A containing $1 \%$ Tx100 similar to sample preparation for Sei1-Ldb16 protein purification. Solubilized membranes (typically in volumes of $900 \mu \mathrm{l}$ ) were centrifuged again for $1 \mathrm{~h}$ at $125,000 \mathrm{~g}, 4^{\circ} \mathrm{C}$ and $500 \mu \mathrm{l}$ were filtered in $0.2-\mu \mathrm{m}$ filters and analyzed by size-exclusion chromatography on a Superose 6 Inc column as described above, followed by SDS-PAGE, western blot and detection of myc tag using anti-myc monoclonal 9E10 (Thermo Fisher Scientific, diluted 1:10,000) and the secondary antibodies anti-mouse-HRP and anti-rabbit-HRP (Santa Cruz Biotechnology, diluted 1:10,000).

Immuno-precipitation. Solubilized membrane extracts in 1\% Triton X-100 from $25 \mathrm{ml}$ of YPD cultures were prepared as described above in a total volume of $1.2 \mathrm{ml}$ and were added to $250 \mu \mathrm{l}$ of anti-myc agarose slurry (Thermo Fisher Scientific) in 2-ml tubes. After incubation for $1 \mathrm{~h}, 4^{\circ} \mathrm{C}$ on a nutator, beads were washed with 
$2 \times 1 \mathrm{ml}$ of buffer $\mathrm{A}+0.01 \%$ Triton X-100. Bound proteins were eluted by addition of $50 \mu \mathrm{l}$ of Laemmli buffer and incubation for $30 \mathrm{~min}$ at $95^{\circ} \mathrm{C}$. Samples were analyzed by SDS-PAGE and western blot.

EM sample preparation and data acquisition. Negative-stained samples were prepared as described in ref. ${ }^{37}$ and imaged on a Tecnai T12 microscope (Thermo Fisher Scientific) equipped with 4,000 ×4,000 CCD camera (UltraScan 4000; Gatan).

Cryo-EM samples were concentrated to roughly $3.5 \mathrm{mg} \mathrm{ml}^{-1}$ in $100-\mathrm{kDa}$ filters, and $2.5 \mu \mathrm{l}$ of sample was added to $30 \mathrm{~s}$ glow discharged Quantifoil holey carbon grids (Cu R1.2/1.3; 400 mesh), blotted with Whatman no. 1 filter paper with roughly $100 \%$ humidity and plunge frozen in liquid ethane using a Vitrobot Mark IV (Thermo Fisher Scientific). Images were collected on a Titan Krios electron microscope (Thermo Fisher Scientific): for details, see Table 1.

EM data processing. Cryo-EM data processing was carried out as described previously ${ }^{18}$. Briefly, images were drift corrected by MotionCor2 (ref. ${ }^{38}$ ) and binned $3 \times 3$ by Fourier cropping to a pixel size of $2.475 \AA$. Defocus values were determined using CTFFIND4 (ref. ${ }^{39}$ ) and motion-corrected sums without dose-weighting Motion-corrected sums with dose-weighting were used for all other steps of imaging processing. After particle picking, two-dimensional classification of selected particles was performed in samclasscas.py (SAMUEL scripts, SamViewer). Initial 3D models of a cylindrical density matching the overall Fld1/Seil complex dimension were generated using SPIDER to perform the initial 3D classification. 3D classification and refinement were performed in Relion 3.0 (ref. ${ }^{40,41}$ ) initially without application of symmetry. After the first rounds of $3 \mathrm{D}$ classification without symmetry on binned particles, the second round of classification was performed on selected particles without binning. This step was followed by global refinement on selected particles with $\mathrm{C}_{10}$ symmetry without subtraction of the micelle. Afterward, the refined particle stack underwent symmetry expansion with $\mathrm{C}_{10}$, and was further classified without global angle search (nonalignment classification). In this and the following steps, the density model from previous refinement result was used as reference. For the final round of refinement $C_{5}$ symmetry was imposed to generate the cryo-EM map of Seil showing signal of the TM region. The final EM density map was sharpened by application of $-75 B$ factor with the filtered resolution of $3.75 \AA$ by the program bfactor.exe ${ }^{42}$. Local resolution variation of EM density maps was calculated in ResMap v.1.1.4 (ref. ${ }^{43}$ ).

Model building and refinement. Seipin density maps in MRC/CCP4 format were converted to structure factors MTZ format in PHENIX suite ${ }^{44}$. Models were built manually in $\mathrm{COOT}^{45}$ starting from the high-resolution region in the ER-lumenal region, and iteratively refined in PHENIX real-space refinement procedure, followed by visual inspection and manual refinement in COOT. The TM segments (residues 25-46; 234-258) of conformation A were manually built. Other parts of the TM segments of conformations A and B were modeled as described below.

Molecular modeling of the TM helices. The trRosetta ${ }^{27}$ neural network was run on the full-length sequence to generate 2,003 aligned sequences. Filtering by $90 \%$ maximum pairwise sequence identity and $50 \%$ minimum sequence coverage yielded 921 sequences, which were used to derive pairwise constraints across the whole structure. The trRosetta constraints were input alongside density data to the Rosetta comparative modeling (RosettaCM) ${ }^{46}$. We leveraged the manually built model from residues $25-258$ in the A conformation and 49-233 in the B conformation as starting models in this pipeline. For each conformer, 10,000 modeling trajectories were sampled and the top models selected by Rosetta showed good agreement with the density. These conformers were input as the asymmetric unit in Rosetta symmetric refinement. $\mathrm{C}_{5}$ symmetry was used to generate the final 10-mer model.

Terbinafine growth assays. Yeast strain BY4741 sei1 $\Delta$ was transformed with plasmids expressing seipin constructs from ADH1 promoters and C-terminal GFP tag by selection on synthetic medium without uracil. Cells were grown to an early stationary phase for $16-24 \mathrm{~h}$ in $3-\mathrm{ml}$ cultures in synthetic medium without uracil $+2 \%$ dextrose. $\mathrm{OD}_{600}$ was determined, and cells diluted to OD of 0.25 . Serial 1:5 dilutions in sterile water were performed in 96-well plates, and $3 \mu \mathrm{l}$ were spotted onto plates with or without $100 \mu \mathrm{g} \mathrm{ml}^{-1}$ terbinafine (Sigma-Aldrich). Plates were imaged after $3-7$ days of incubation at $30^{\circ} \mathrm{C}$.

Fluorescence microscopy. Here, $1 \mu$ BODIPY $\left(\mathrm{C}_{9} \mathrm{H}_{7} \mathrm{BN}_{2} \mathrm{~F}_{2}\right)$ 493/503 from a $1 \mathrm{mg} \mathrm{ml}^{-1}$ stock in dimethylsulfoxide (stored in the dark) was added to $1 \mathrm{ml}$ of culture in a $1.5-\mathrm{ml} \mathrm{microfuge} \mathrm{tube} \mathrm{and} \mathrm{incubated} \mathrm{on} \mathrm{a} \mathrm{rocker} \mathrm{(dark)} \mathrm{for} 30 \mathrm{~min}$, centrifuged for $1 \mathrm{~min}$ at $2,000 \mathrm{~g}$ and $950 \mu \mathrm{l}$ of the supernatant removed. Cells were resuspended in the remaining media and $1.7 \mu \mathrm{l}$ of the cell suspension applied to a slide for microscopy. Alternatively, cells from $3 \mathrm{ml}$ of culture were centrifuged $(20 \mathrm{~s}$, $18,000 \mathrm{~g}$ ), resuspended in $50 \mu \mathrm{l}$ of synthetic medium $+5 \mu \mathrm{l}$ of 1:250 diluted autodot dye (Abcepta) and incubated as described above.

The microscope hardware, and image acquisition and projections from $z$-stacks were as reported previously ${ }^{47}$, except the $z$-stack consisted of 25 images taken $0.35 \mu \mathrm{m}$ apart, and Slidebook v.6.0.4 (Intelligent Imaging Innovations) was used. Alternatively, cells were imaged on a Nikon Eclipse Ti inverted microscope equipped with CSU-X1 spinning-disc confocal scan head (Yokogawa), 405-, 488and 561-nm laser lines, $100 \times$ Apochromat total internal reflection fluorescence 1.4 numerical aperture objective (Nikon), Zyla 4.2 Plus sCMOS or iXon897 electron-multiplying charged-coupled device cameras (Andor) and NIS Elements AR software (Nikon).

Cell culture. Typically, seipin protein expression was determined on cultures that were also subjected to fluorescence microscopy to determine number and size of LD. A colony from each strain was inoculated into $5 \mathrm{ml}$ of synthetic complete dextrose (SCD)-defined medium ${ }^{14}$ and incubated for 20-24h in a rotary shaker, then back-diluted to $\mathrm{OD}_{600}$ of 0.1 per $\mathrm{ml}$ into $50 \mathrm{ml}$ of SCD and incubated for $24 \mathrm{~h}$. The culture was then immediately processed for both fluorescence microscopy and immunoblotting.

Cell segmentation. To facilitate cell segmentation in brightfield images, a deep learning pipeline for automatic instance segmentation was implemented, mostly following ref. ${ }^{48}$. In short, we trained a convolutional neural network to jointly make three pixel-wise predictions: a seed map, a scalar bandwidth and two-dimensional spatial embeddings, which were used to differentiate cells. After adding the pixel coordinates, the spatial embeddings should be constant over each cell, while different cells should have distinct embeddings.

To train the neural network, the above condition was encouraged in an indirect manner. For every cell, the average embedding vector was computed and a soft mask was grown, using a Gaussian kernel of the average predicted bandwidth over that cell in the embedding space. Using a loss for binary classification, these soft masks were driven to match the binary ground-truth masks of the cells. In this, we deviated from ref. ${ }^{48}$ and used the Dice-Loss algorithm ${ }^{49}$, which works well with the class imbalance between foreground and background.

In the inference procedure, an instance segmentation was inferred using an iterative algorithm ${ }^{48}$. The pixel with the highest score in the seed map was selected as the seed, and all pixels whose spatial embeddings are sufficiently close to the embedding of the seed pixel were clustered as a predicted instance. This process was repeated, conditioning the selection of the seed pixel to the not yet assigned regions, until all foreground pixels (that is, pixels with a seed score over 0.5 ) were assigned to an instance.

As postprocessing, we filtered out predicted instances whose size falls below a threshold of 300 pixels as well as those that touch the image border. Finally, the convex hulls of the predicted segments were converted to a list of FIJI/ImageJ regions of interest, on which we carried out the downstream analysis.

To generate the necessary ground-truth data, 18 images were annotated. For each living cell (that is, cells without dense cytoplasm) in those images, an ellipse was drawn in FIJI/ImageJ, which was converted to a pixel-wise mask for that instance. These masks were then combined to generate the label images required to train the neural network. Of the 18 images, we used 14 for training and four for validation.

As the architecture of our model, we choose a variant of U-Net ${ }^{50}$ with additional residual connections ${ }^{51}$ at every scale in both the encoding and decoding branches. Each convolution was followed by a batch normalization layer ${ }^{52}$.

The network was trained on $1,024 \times 1,024$-pixel crops of the annotated images, which were randomly flipped and rotated to augment the training data and thereby combat overfitting. We used the Adam optimizer ${ }^{53}$ with a learning rate of $10^{-4}$. The model was trained on a single graphics card, while predictions were computed on the CPU to simplify deployment. Finally, the calculated cells perimeters for each field, which were converted to ImageJ region of interest files.

Fluorescence image quantification. Seipin loss-of-function results in fewer and larger 'supersized' droplets (or aggregates of small droplets). The number of supersized droplets in seipin mutants are enhanced in $p \ln 1 \Delta$ strains $^{47}$ and for this reason, most experiments with seipin mutants were performed in a $\operatorname{pln} 1 \Delta$ background.

The heterogeneity of LD sizes, number and tendency of LDs to cluster presented a challenge for automated LD counting. An ImageJ routine was written to count particles per cell (regions of interest) in each field iteratively at decreasing lower thresholds (upper threshold was set at maximal), starting at 20,000 in 2,000 increments and ending at 2,000. (At the 20,000 threshold only the largest droplets were counted, whereas at 2,000 very dim droplets were counted while the point-spread function of larger ones in clusters merged.) The droplet number for each cell was the maximal particle count over the threshold range. This correlated well to droplet counts determined visually except the dynamic range was attenuated, as very dim droplets were counted (increasing the count), but LDs in clusters of droplets were not resolved (decreasing the count). However, the relative values among strains corresponded well to the visual counts (not shown).

Scoring cells with supersized droplets was performed by counting BODIPY-stained particles per cell at 10,000 lower threshold in ImageJ with an area (point-spread function at this threshold) of greater than $0.5 \mu \mathrm{m}^{2}$.

Statistical analysis. All experiments were carried out in three independent repeats, unless noted otherwise in figure legends. For analysis of LD phenotypes, three microscope fields for each strain in each experiment, each typically with 200 cells, 
were analyzed as described for droplets per cell and cells containing LDs over $0.5 \mu \mathrm{m}^{2}$. The mean value was obtained from the three fields and represented as a single data point on graphs. To determine significant differences among strains, a one-way analysis of variance (ANOVA) was performed on the mean values with strains in each experiment linked, followed by the Holm-Sidak test on preselected pairs of strains; GraphPad Prism v.9 software was used for the graphs and analysis: ${ }^{\star} P<0.05 ;{ }^{* *} P<0.01$ and ${ }^{* *} P<0.001$.

Cell lysates and immunoblots. Here, $30 \mathrm{OD}_{600}$ units of cells were removed, centrifuged at $3,000 \mathrm{~g}$ for $5 \mathrm{~min}$ and the pellets washed in $25 \mathrm{ml}$ of $\mathrm{H}_{2} \mathrm{O}$. Washed cell pellets were resuspended in $1 \mathrm{ml}$ of $\mathrm{H}_{2} \mathrm{O}$, transferred to microfuge tubes and centrifuged at top speed $(18,000 \mathrm{~g})$ in a microfuge for $1 \mathrm{~min}$. Pellets were resuspended in $450 \mu \mathrm{l}$ of $\mathrm{H}_{2} \mathrm{O}$ and chilled on ice. Then, $50 \mu \mathrm{l}$ of $100 \%$ trichloroacetic acid was added, and the tubes vortexed and then allowed to remain on ice for $15 \mathrm{~min}$. The suspension was centrifuged at $4^{\circ} \mathrm{C}$ for $5 \mathrm{~min}$ and the supernatant was removed. Pellets were centrifuged for $15 \mathrm{~s}$, and the remaining supernatant was pipetted off. After that, $300 \mu \mathrm{l}$ of $2 \times$ Laemmli sample buffer was added, and the cells were resuspended by vortexing. To turn the suspension from yellow (from the residual acid) to blue, $4-5 \mu \mathrm{l}$ of $5 \mathrm{~N} \mathrm{NaOH}$ was added. Then, $250 \mathrm{mg}$ of acid-washed glass beads were added, and the suspensions were subjected to three 1-min pulses in a mini-bead beater (Biospec Products) in the cold room to lyse the cells. Samples were placed in a boiling water bath for $5 \mathrm{~min}$ and chilled. Leaving the beads behind, lysates were transferred to fresh tubes and centrifuged for $5 \mathrm{~min}$ at top speed. Supernatants were collected and used for immunoblotting.

The protein concentrations of the lysates were determined by an amido black filtration assay ${ }^{54}$ with Fraction V BSA (Sigma) for a standard curve. Then $20 \mu \mathrm{g}$ of cell lysates were added to $10 \%$ SDS gels for polyacrylamide electrophoresis and detection of seipin; in parallel, $2 \mu \mathrm{g}$ of lysates were run out for detection of G6PDH. Proteins were electroblotted from gels onto nitrocellulose. Blots were treated for $1 \mathrm{~h}$ or overnight with LI-COR PBS Blocking Buffer (diluted 1:4 in tris-buffered saline (TBS) and Polysorbate 20 (TBST)) and then subjected to first and second antibody, with three 5-min washes with TBST after each, before visualization on a LI-COR Biotechnology Odyssey Infrared Imaging System (Lincoln, NE) and quantification of bands using Image Studio v.5.2.5 (LI-COR).

Antibodies for immunoblots: primary antibodies included anti-myc monoclonal 9E10 (Thermo fisher, diluted 1:10,000) or anti-G6PDH (Sigma, diluted 1:20,000). Secondary antibodies included goat anti-mouse and goat anti-rabbit IRDye antibodies, used according to the manufacturer (LI-CORE)

Reporting Summary. Further information on research design is available in the Nature Research Reporting Summary linked to this article.

\section{Data availability}

Coordinates of the oligomeric structure of yeast seipin and the corresponding electron density map have been deposited to Protein Data Bank accession code PDB 7RSL and Electron Microscopy Data Bank accession code EMD-24674, respectively. Source data are provided with this paper.

\section{Code availability}

For cell segmentation, software was downloaded from Github and installed on Macintosh computers; it is available at https://github.com/hci-unihd/YeastCellSeg for public use.

\section{References}

35. Thomas, B. J. \& Rothstein, R. Elevated recombination rates in transcriptionally active DNA. Cell 56, 619-630 (1989).

36. Janke, C. et al. A versatile toolbox for PCR-based tagging of yeast genes: new fluorescent proteins, more markers and promoter substitution cassettes. Yeast 21, 947-962 (2004).

37. Booth, D.S., Avila-Sakar, A. \& Cheng, Y. Visualizing proteins and macromolecular complexes by negative stain EM: from grid preparation to image acquisition. J. Vis. Exp. https://doi.org/10.3791/3227 (2011).

38. Zheng, S. Q. et al. MotionCor2: anisotropic correction of beam-induced motion for improved cryo-electron microscopy. Nat. Methods 14, 331-332 (2017).

39. Rohou, A. \& Grigorieff, N. CTFFIND4: fast and accurate defocus estimation from electron micrographs. J. Struct. Biol. 192, 216-221 (2015).

40. Scheres, S. H. RELION: implementation of a Bayesian approach to cryo-EM structure determination. J. Struct. Biol. 180, 519-530 (2012).

41. Scheres, S. H. A Bayesian view on cryo-EM structure determination. J. Mol. Biol. 415, 406-418 (2012).

42. Lyumkis, D., Brilot, A. F., Theobald, D. L. \& Grigorieff, N. Likelihood-based classification of cryo-EM images using FREALIGN. J. Struct. Biol. 183, 377-388 (2013).

43. Kucukelbir, A., Sigworth, F. J. \& Tagare, H. D. Quantifying the local resolution of cryo-EM density maps. Nat. Methods 11, 63-65 (2014).
44. Adams, P. D. et al. PHENIX: building new software for automated crystallographic structure determination. Acta Crystallogr. D. Biol. Crystallogr. 58, 1948-1954 (2002)

45. Emsley, P., Lohkamp, B., Scott, W. G. \& Cowtan, K. Features and development of Coot. Acta Crystallogr. D. Biol. Crystallogr. 66, 486-501 (2010).

46. Song, Y. et al. High-resolution comparative modeling with RosettaCM. Structure 21, 1735-1742 (2013).

47. Gao, Q. et al. Pet10p is a yeast perilipin that stabilizes lipid droplets and promotes their assembly. J. Cell Biol. 216, 3199-3217 (2017).

48. Agrawal, H. et al. nocaps: novel object captioning at scale. In Proc. IEEE/CVF International Conference on Computer Vision (ICCV) 8947-8956 (IEEE, Seoul, 2019).

49. Milletari, F., Navab, N. \& Ahmadi, S.-A. V-net: fully convolutional neural networks for volumetric medical image segmentation. In Proc. 2016 Fourth International Conference on 3D Vision (3DV) 565-571 (IEEE, Stanford, 2016).

50. Ronneberger, O., Fischer, P. \& Brox, T. U-Net: Convolutional Networks for Biomedical Image Segmentation 234-241 (Springer International Publishing, 2015).

51. He, K., Zhang, X., Ren, S. \& Sun, J. Deep residual learning for image recognition. In Proc. 2016 IEEE Conference on Computer Vision and Pattern Recognition (CVPR) 770-778 (IEEE, Las Vegas, 2016).

52. Ioffe, S. \& Szegedy, C. Batch normalization: accelerating deep network training by reducing internal covariate shift. In ICML'15: Proceedings of the 32nd International Conference on International Conference on Machine Learning 448-456 (PMLR, Lille, 2015).

53. Kingma, D. P. \& Ba, J. Adam: a method for stochastic optimization. Preprint at https://arxiv.org/abs/1412.6980 (2014).

54. Schaffner, W. \& Weissmann, C. A rapid, sensitive, and specific method for the determination of protein in dilute solution. Anal. Biochem. 56, 502-514 (1973).

55. Notredame, C., Higgins, D. G. \& Heringa, J. T-Coffee: a novel method for fast and accurate multiple sequence alignment. J. Mol. Biol. 302, 205-217 (2000).

56. Robert, X. \& Gouet, P. Deciphering key features in protein structures with the new ENDscript server. Nucleic Acids Res. 42, W320-W324 (2014).

57. Kelley, L. A., Mezulis, S., Yates, C. M., Wass, M. N. \& Sternberg, M. J. The Phyre2 web portal for protein modeling, prediction and analysis. Nat. Protoc. 10, 845-858 (2015).

58. Tunyasuvunakool, K. et al. Highly accurate protein structure prediction for the human proteome. Nature 596, 590-596 (2021).

\section{Acknowledgements}

We thank S. Kim and G. Voth (Department of Chemistry, University of Chicago) for critical discussions, K. Brock and D. Marks (HMS, Systems Biology) for advice on evolutionary coupling analyses and C.-W. Wang (Institute of Plant and Microbial Biology, Academia Sinica, Taipei City) for kindly sharing the Ldb16 antibody. We thank D. Moazed (HMS, Cell Biology) for sharing of equipment, S. Sterling, R. Walsh and Z. Li at the Harvard cryo-EM center for EM data collection, J. Reus, N. Paul, A. Gan and D. Chukwuma for generating and phenotyping several yeast strains and G. Howard for editorial assistance. This work was supported by National Institutes of Health (NIH) grant no. R01GM124348 (to R.V.F.), NIH grant no. R01GM084210 (to J.M.G.), a German Research Foundation (DFG) fellowship no. AR1164/1-1 (to H.A.) and an American Heart Association postdoctoral fellowship no. 18POST34030308 (to X.S.). T.C.W. is an investigator of the Howard Hughes Medical Institute.

\section{Author contributions}

H.A., F.A.H., M.L., J.M.G., R.V.F. and T.C.W. designed and supervised the project. H.A., X.S., B.F., X.C., R.R. and J.M.G. carried out and analyzed experiments. H.A. and X.S. performed cryo-EM analysis and model building. C.A. and F.D. carried out machine learning calculations and modeling on the TM helices. H.A. created figures and H.A., J.G., R.V.F. and T.C.W. wrote the manuscript, which all authors read and edited.

\section{Competing interests}

The authors declare no competing interests.

\section{Additional information}

Extended data are available for this paper at https://doi.org/10.1038/s41594-021-00718-y.

Supplementary information The online version contains supplementary material available at https://doi.org/10.1038/s41594-021-00718-y.

Correspondence and requests for materials should be addressed to Joel M. Goodman, Robert V. Farese or Tobias C. Walther.

Peer review information Nature Structural and Molecular Biology thanks Abdou Rachid Thiam and the other, anonymous, reviewer(s) for their contribution to the peer review of this work. Florian Ullrich was the primary editor on this article and managed its editorial process and peer review in collaboration with the rest of the editorial team.

Reprints and permissions information is available at www.nature.com/reprints. 
a

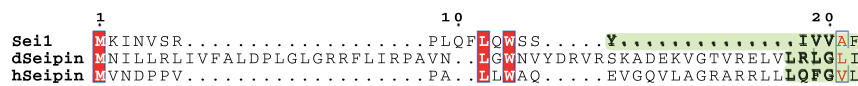

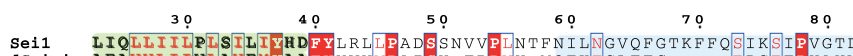

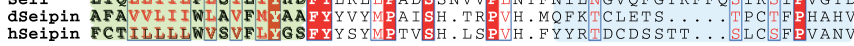

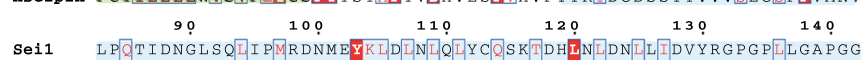

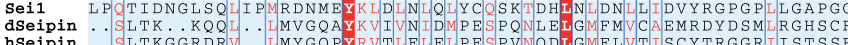
hSeipin ..
150

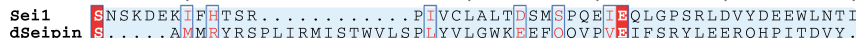

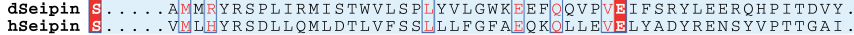

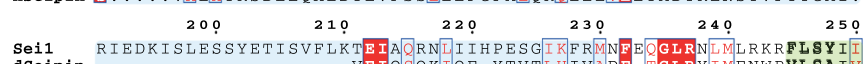

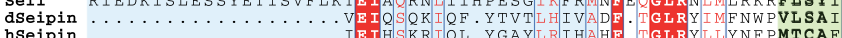
$260 \quad 270$

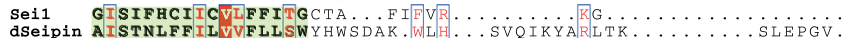

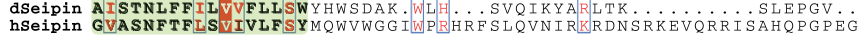
Sei1 28 O

dSeepin IHSKASSLRDDDDL ........ VAYSDKSDIADVGGDTLSDVDAD ........ DLV

b

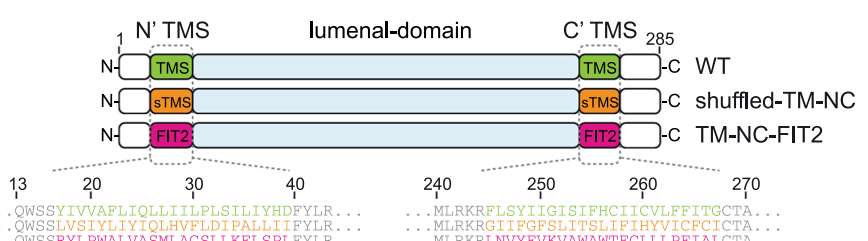

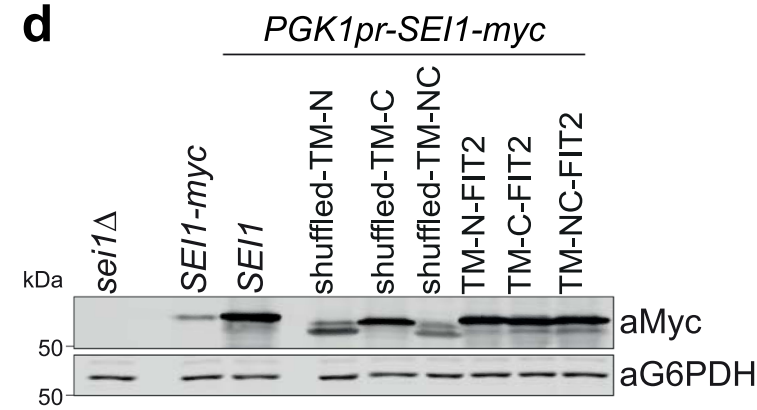

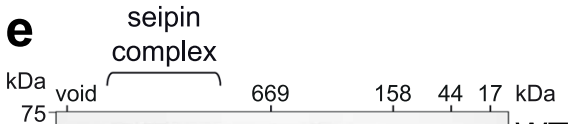

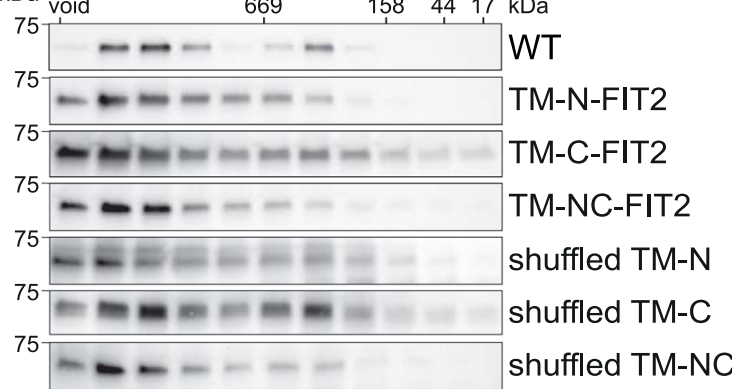

C
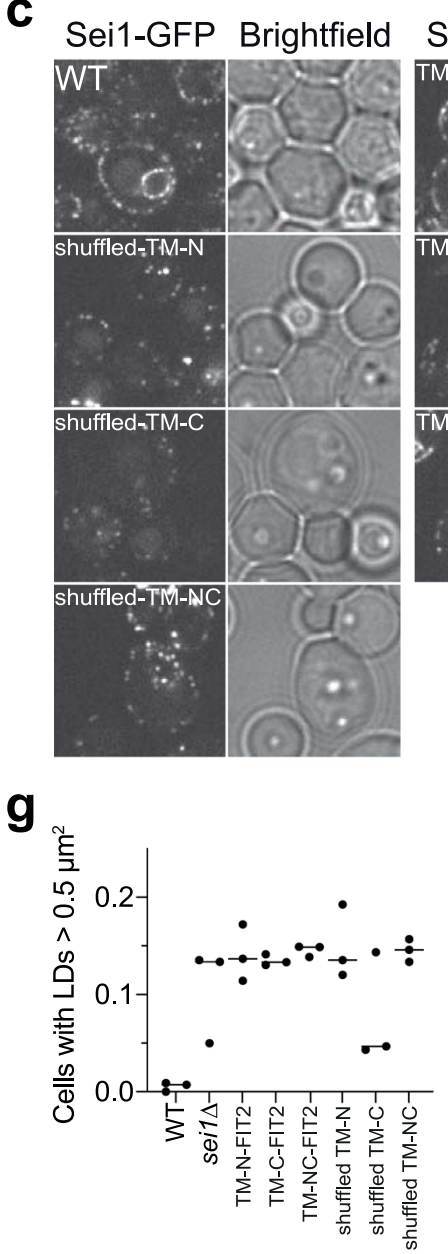

f

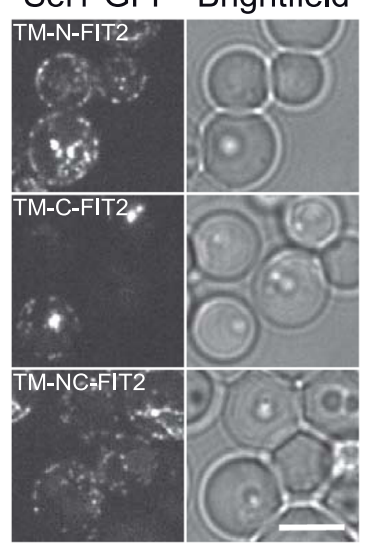

h

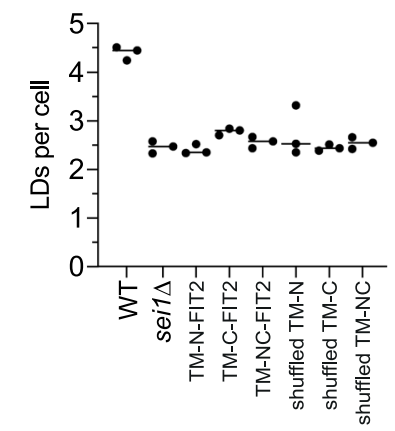

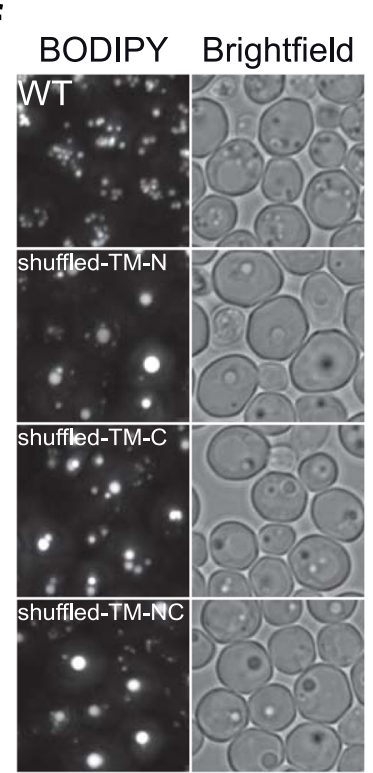

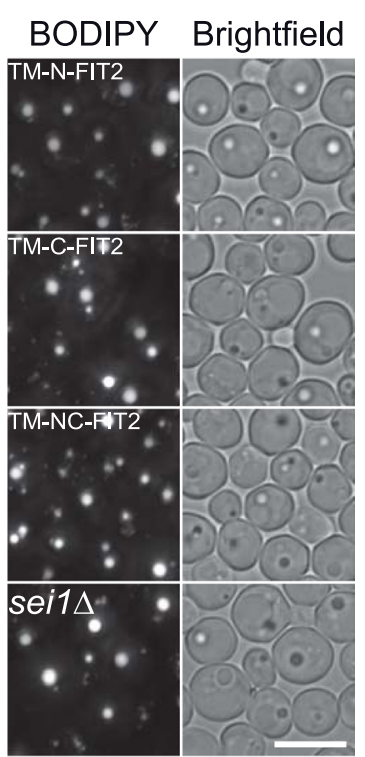

i

sei1ム

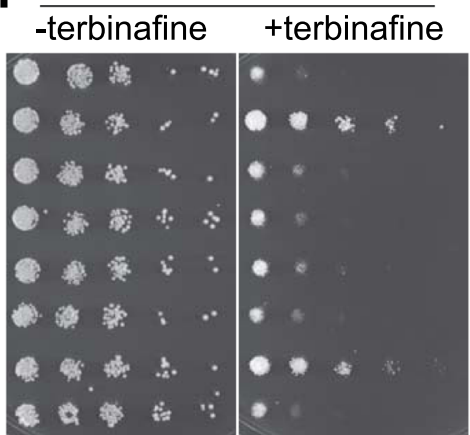

empty vector
WT
TM-N-FIT2
TM-C-FIT2
TM-NC-FIT2
shuffled TM-N
shuffled TM-C
shuffled TM-NC

Extended Data Fig. 1 | See next page for caption. 
Extended Data Fig. 1 | Transmembrane segments of seipin are conserved and required for function. (a) Sequence alignment of yeast (Saccharomyces cerevisiae) seipin (Sei1) protein sequence with Drosophila melanogaster (dseipin) and human seipin (hseipin) in T-COFFEE ${ }^{55}$, plotted in ESPript 3.056. Identical residues are colored in red boxes, red characters and blue framed residues indicate similarity in a group or across groups, respectively. TM segments are colored in green and lumenal domains in cyan background similar to overview in b. (b) Overview of mutants analyzed in this figure. Detailed sequence information yeast seipin constructs is shown at the bottom for WT (green), shuffled-TMS (orange) and FIT2-TMS (pink). TMS, transmembrane segment. (c) Localization of seipin WT and mutant constructs expressed from plasmids in seit $\Delta$ cells. Size bar $=5 \mu \mathrm{m}$. (d) Expression level of WT and mutant constructs tagged with C-terminal 13xmyc. SEI1-myc indicates expression level from endogenous promoter. (e) Transmembrane mutants form normal oligomers in detergent extracts. Size-exclusion analysis of membrane extract from cells expressing SEl7-13xmyc or indicated mutants from the endogenous locus driven by integrated PGK1 promoter. Representative immunoblots of two biologically independent experiment repeats are shown. (f) Analysis of LD morphology using BODIPY staining. Seipin mutants with C-terminal 13xmyc tag were expressed from PGK1 promoter. Size bar $=5 \mu$ m. $(\mathrm{g}, \mathrm{h})$ Quantification of experiment in panel $\mathrm{f} . \mathrm{n}=3$ biologically independent experiments. (i) Growth of indicated mutants on synthetic medium $\pm 100 \mu \mathrm{g} / \mathrm{ml}$ terbinafine. 


\section{a \\ b}
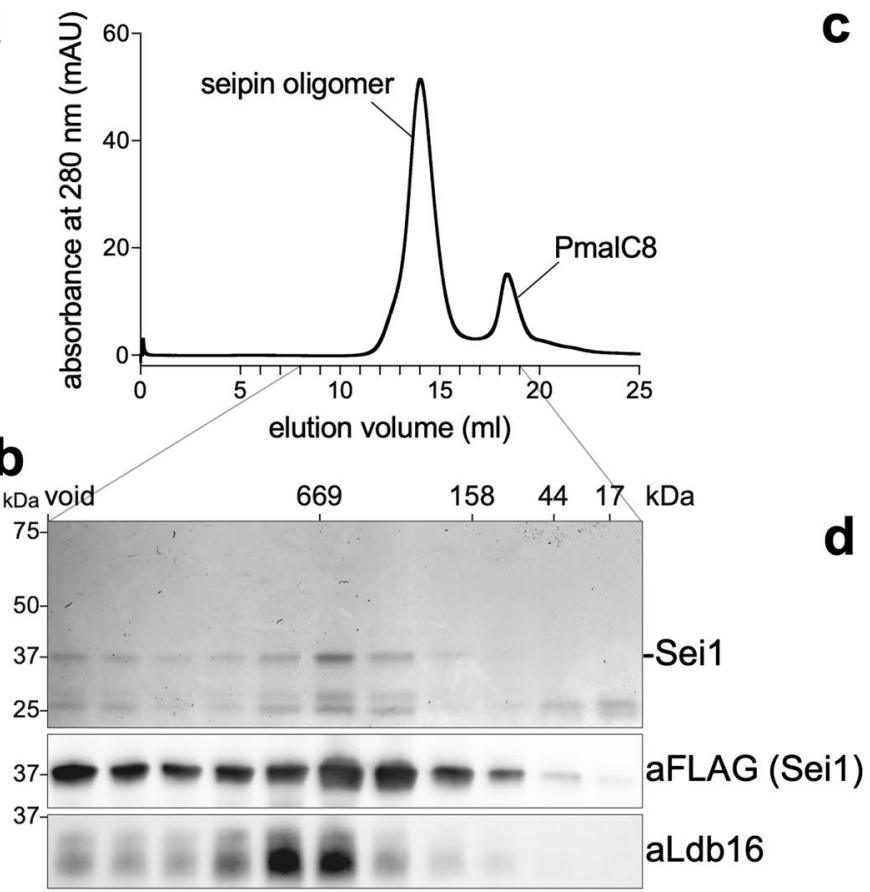

e

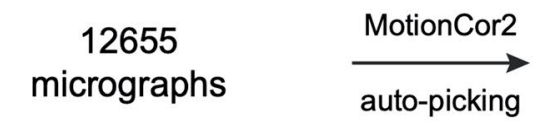

C

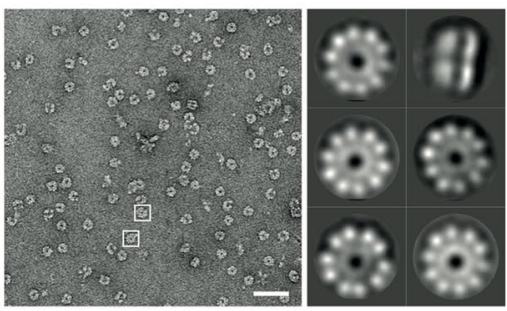

d

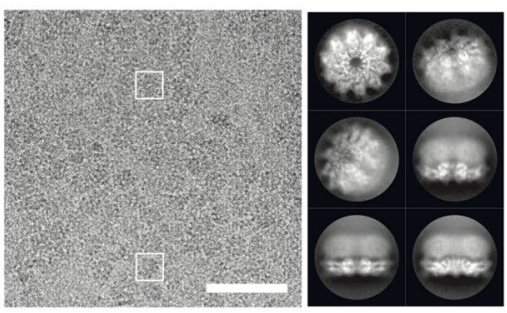

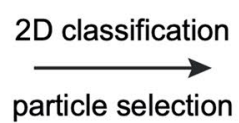

$1.1 \mathrm{M}$ particles $\downarrow$ (no symmetry)

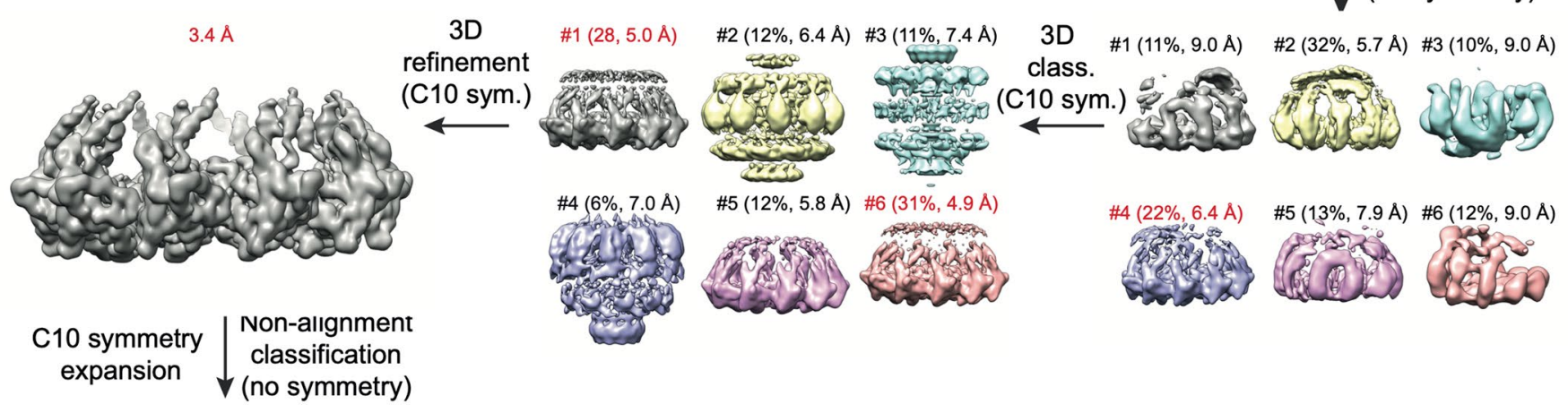

\#1 $(49 \%, 3.8 \AA) \quad \# 2(3 \%, 4.1 \AA) \quad \# 3(3 \%, 3.8 \AA)$
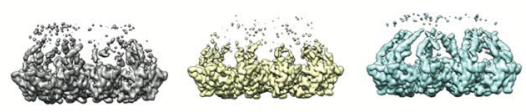

3D

refinement

\#4 (35\%, $3.7 \AA)$

\#5 (6\%, $4.0 \AA) \quad \# 6(3 \%, 4.1 \AA)$

(C5 sym.)
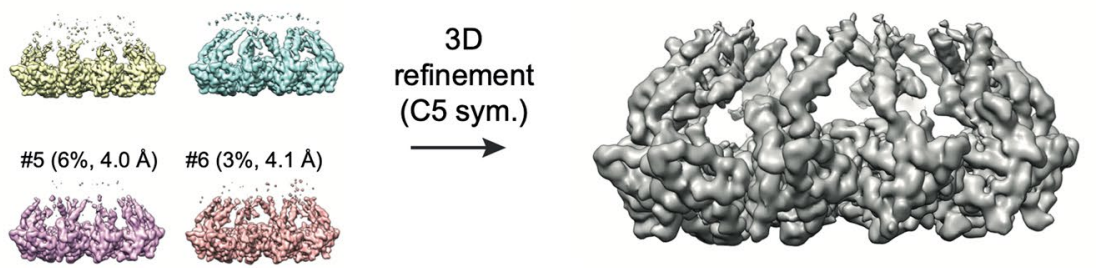

Extended Data Fig. 2 | Purification and cryo-EM image processing of yeast seipin-Ldb16 complex. (a) Sei1-Ldb16 complex was purified from yeast as described in Experimental Procedures. After extraction of the complex in Triton X-100, detergent was subsequently exchanged to digitonin, and finally to PmalC8. The complex was separated by size-exclusion chromatography column in buffer without detergents. (b) Analysis of 1-ml fractions (8-18 ml) after SDS-PAGE by Coomassie Blue staining (top) or Western-blot (bottom). (c) Representative negative stain-EM image of purified complexes shown in a and b. Right side shows 2D class averages. White boxes indicate single oligomers. Size-bar, 500 A. (d) Representative cryo-EM image of purified Sei1-Ldb16 complex. Right side shows 2D class averages. White boxes indicate single oligomers. Size-bar, $500 \AA$. (e) Three-dimensional classification and refinement of cryo-EM particles in Relion 3.0. 
a

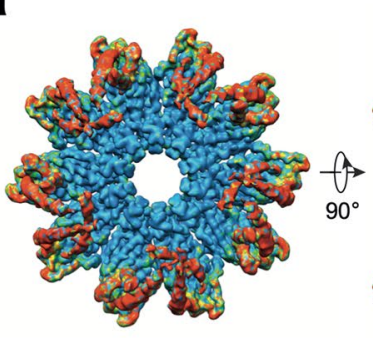

C

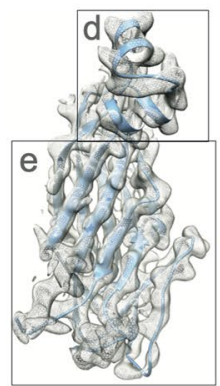

e

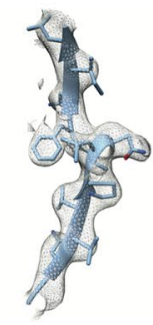

51-61

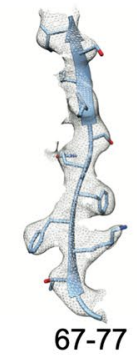

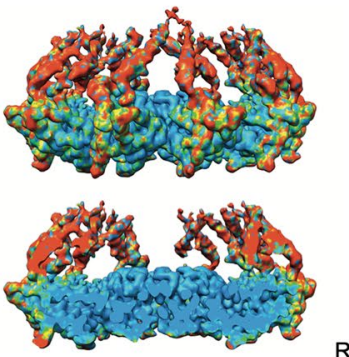

d b

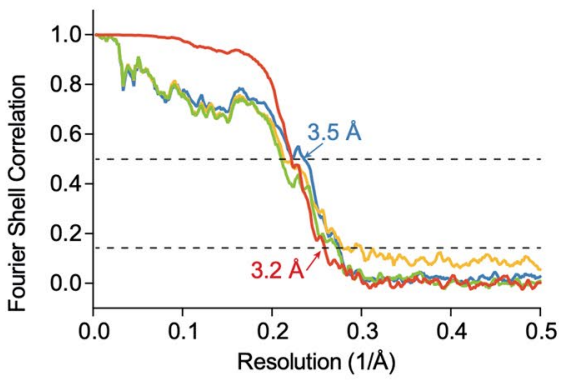

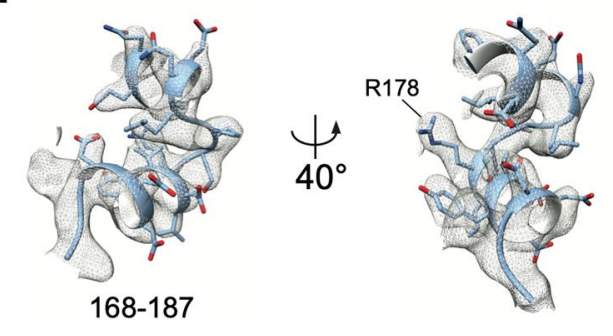
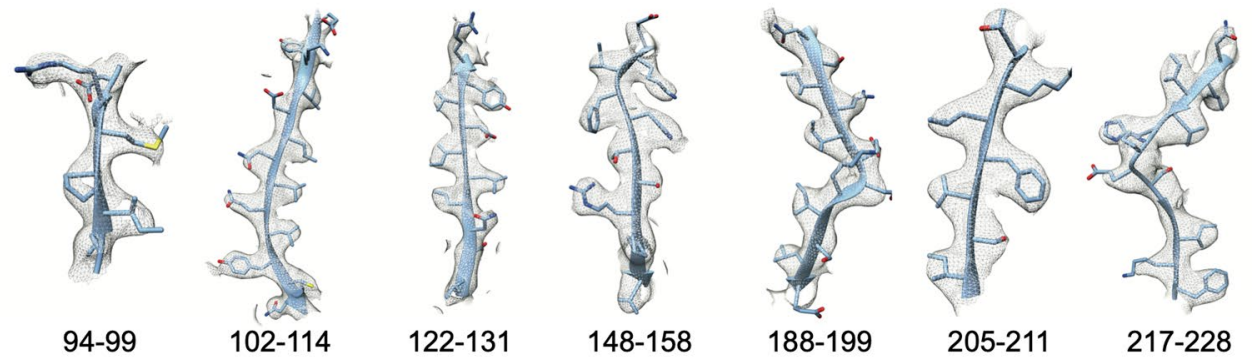

$\mathbf{f}$
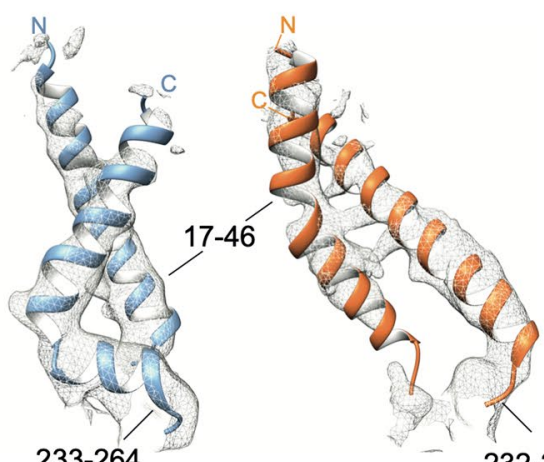

9

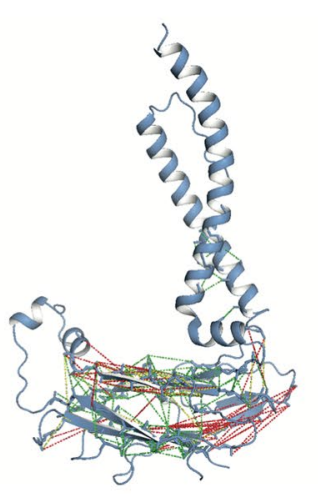

h

232-264

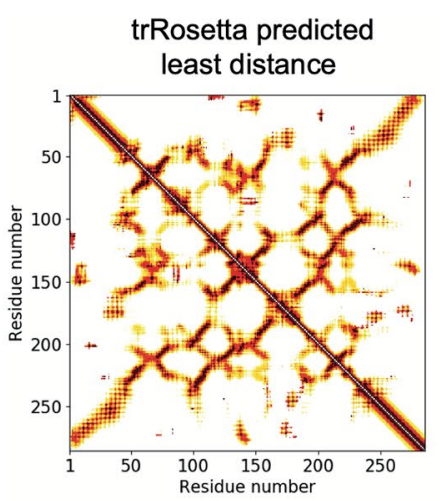

Actual distances in conf. A extended model
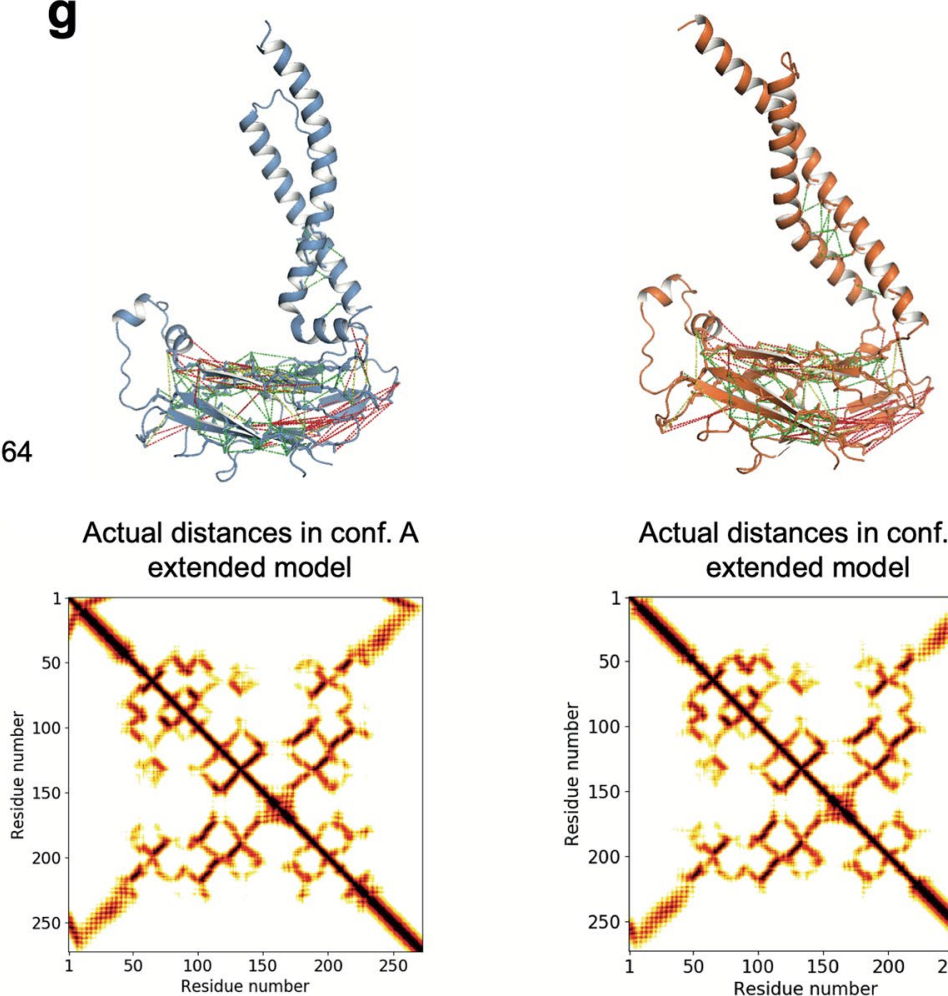

Actual distances in conf. B extended model

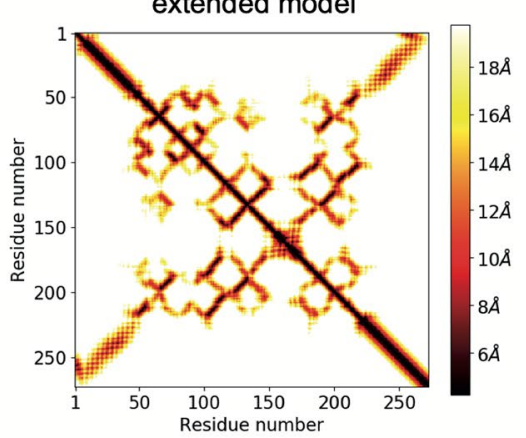

Extended Data Fig. 3 | See next page for caption. 
Extended Data Fig. 3 | Single-particle cryo-EM analysis of Sei1-Ldb16 complex. (a) Local resolution mapped onto EM density map using Resmap ${ }^{43}$ shows differences between lumenal and transmembrane regions of the map. (b) FSC curves: gold-standard FSC curve between the two half maps with indicated resolution at FSC $=0.143$ (red); half-map 1 (green), half-map 2 (orange) and the atomic model refined against half map 1 (blue). (c-e) Superimposed cryo-EM densities from sharpened map with atomic model for central alpha-helices (d) and individual beta-sheets (e). (f) Superimposed cryo-EM densities from unsharpened map with atomic model for TM segments of conformation A (blue) and conformation B (orange). (g) Extended models for conformation $A$ (left) and B (right). Residues at least 10 residues apart in the primary sequence predicted to have beta-carbons interacting within $10 \AA$ distance, with maximal probability and over $70 \%$ probability mass, mapped onto the final model of conformation $A$ (left) and $B$ (right). Green indicates that the actual distance is within $10 \AA$, yellow within $12 \AA$, and red for $>12 \AA$. (h) The predicted and actual distances between beta-carbons of residues in the seipin monomer. The color of each pixel corresponds to the distance in $\AA$ between these atoms. Plotted on the left is the least distance predicted by trRosetta for each pair of CB atoms. In the middle are actual distances in conformations A, and conformation B (right). The trRosetta pipeline correctly predicts interactions between the $\mathrm{N}$ - and C-terminal helices for both conformations (from residues 10-40 and 250-280). 
a

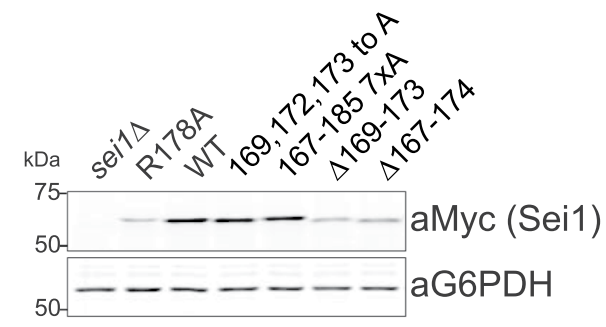

b

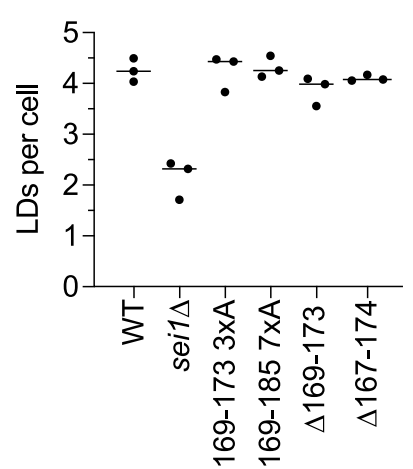

d

\begin{tabular}{|c|c|c|}
\hline \multicolumn{2}{|c|}{$\operatorname{sei1} \Delta$} & \\
\hline -terbinafine & +terbinafine & \\
\hline 00 की & $7 a$ & empty vector \\
\hline 0 o in: & $00 * *$ & WT \\
\hline $00 \%$ : & $0 \cdots *$ & $169,172,173$ to $A$ \\
\hline $000 * 2$ & $0 \circ \cdots$ & 167-185 7xA \\
\hline $00 \leqslant *::$ & $00 * 1$ & $\Delta 169-173$ \\
\hline 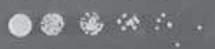 & $00 * 4$ & $\Delta 167-174$ \\
\hline
\end{tabular}

e

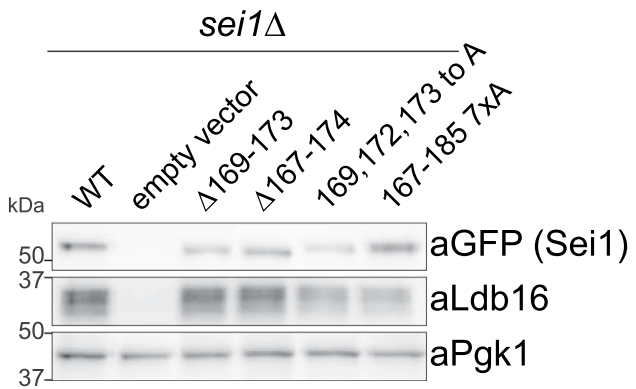

Extended Data Fig. 4 | Mutants in seipin's lumenal central helix retain function in vivo. (a) Western blot analysis of seipin expression level. Cells expressing WT or indicated mutant constructs with C-terminal 13xmyc tag from the endogenous promoter. Sei1 detected with anti-myc antibodies. $(b, c)$ Quantification of images shown in Fig. 2c. $n=3$ biologically independent experiments. (d) Growth of yeast strain sei1 $\Delta$ carrying vectors with C-terminally GFP-tagged SEl1 sequences or empty vector on synthetic medium $\pm 100 \mu \mathrm{g} / \mathrm{ml}$ terbinafine. (e) Western blot analysis of whole-cell-lysates from strains in d using antibodies against GFP to detect seipin, against Ldb16 or Pgk1 as loading control. Representative immunoblots of two biologically independent experiment repeats is shown. 
a

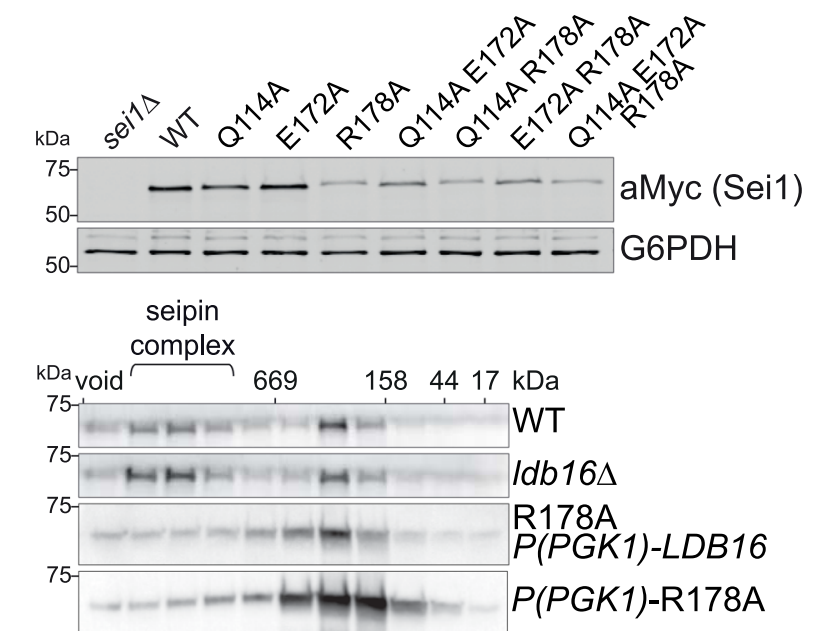

d
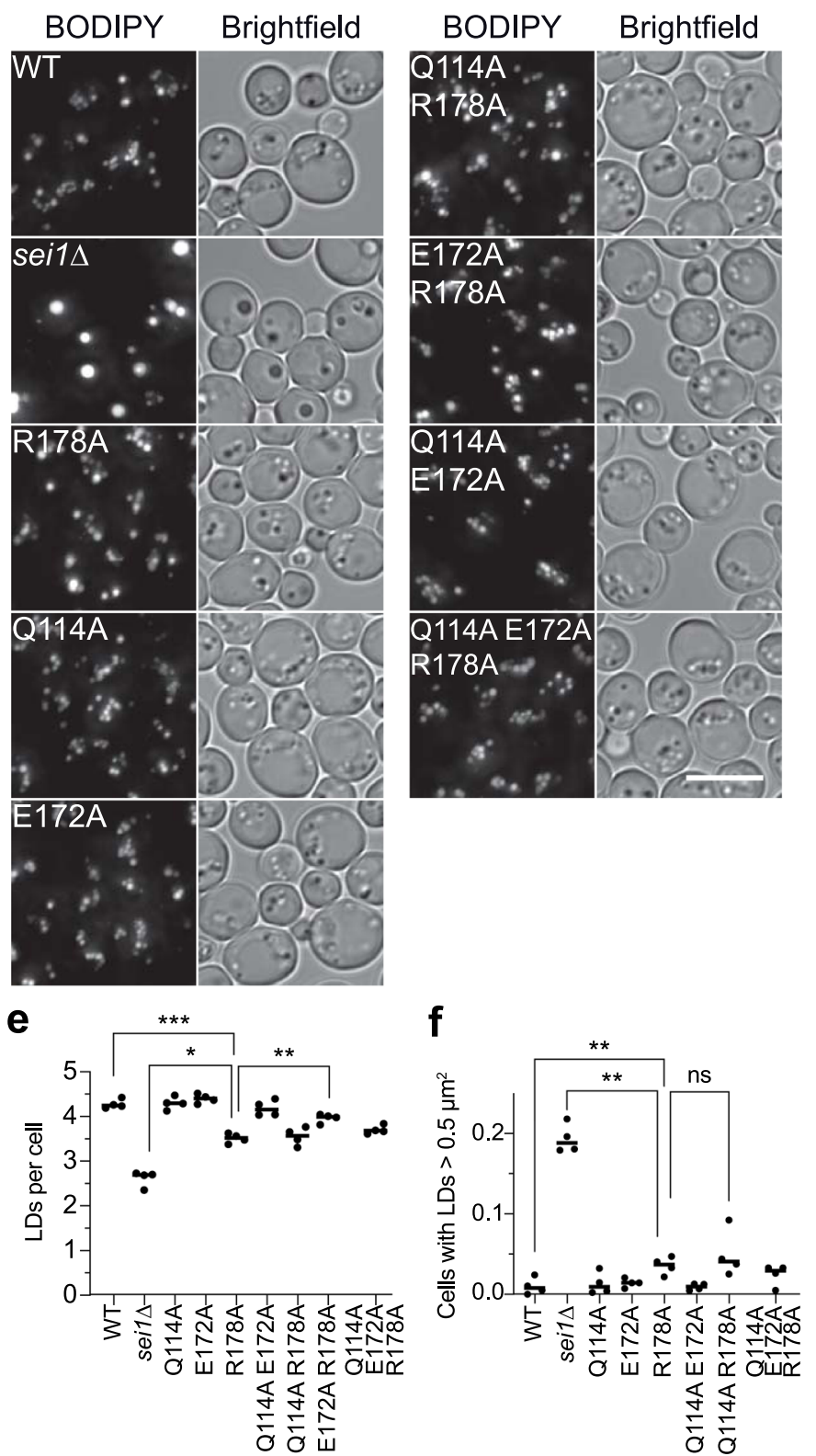

C

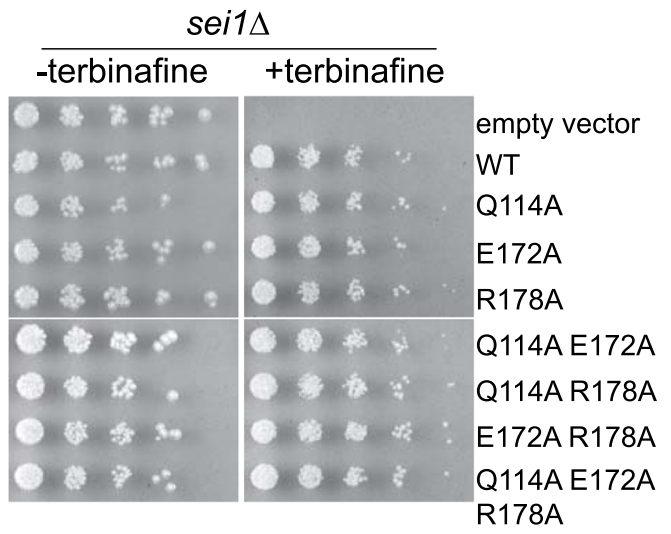

g
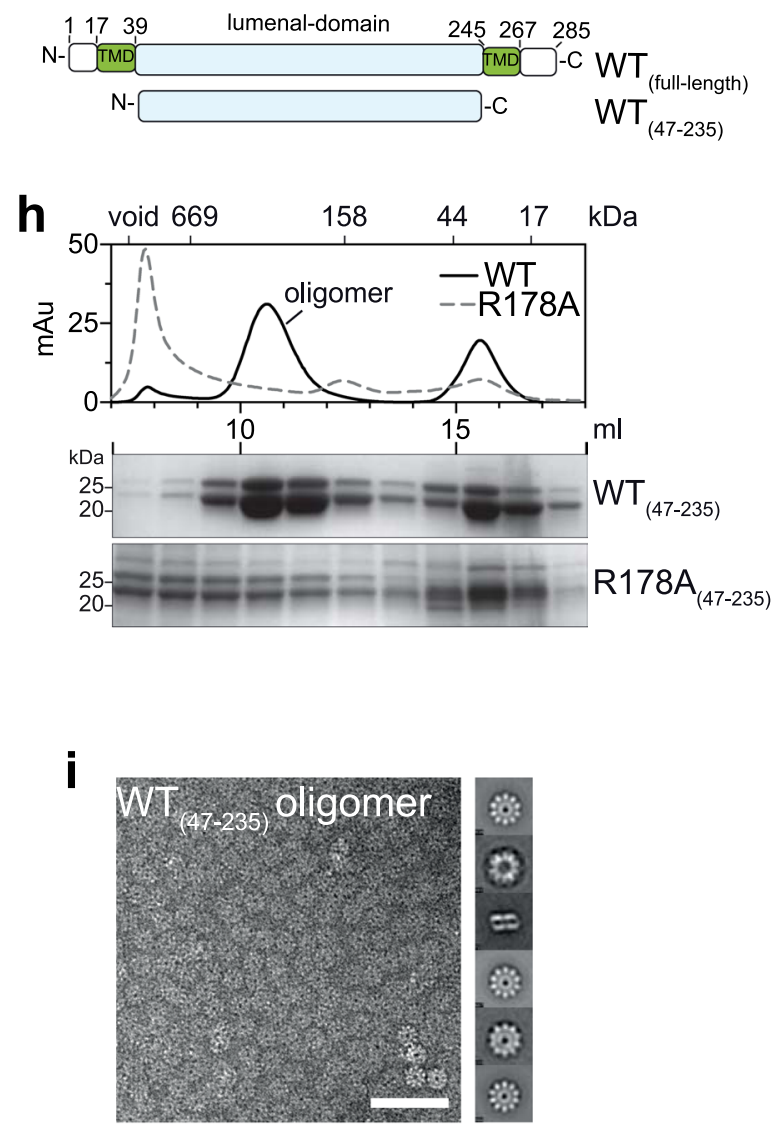

Extended Data Fig. 5 | See next page for caption. 
Extended Data Fig. 5 | Lumenal domain interactions are mediated by R178. (a) Western blot analysis with anti-myc antibodies of lysate from strains expressing WT seipin or indicated point mutations from the endogenous locus with C-terminal 13xmyc tag. (b) Size-exclusion chromatography of Triton X-100 solubilized membrane extracts of indicated strains expressing C-terminal 13xmyc-tagged seipin. Representative immunoblots of two biologically independent experiment repeats is shown. (c) Growth of yeast strain sei1A carrying vectors with C-terminally GFP-tagged SEI1 mutants or empty vector on synthetic medium $\pm 100 \mu \mathrm{g} / \mathrm{ml}$ terbinafine. (d) LD morphology of strains expressing indicated seipin mutants with C-terminal 13xmyc from endogenous locus. Size bar, $5 \mu \mathrm{m},(\mathrm{e}, \mathrm{f})$ Quantification of experiment shown in $\mathrm{d} . \mathrm{n}=4$ biologically independent experiments. Data were analyzed with one-way ANOVA and Holm-Sidak's posthoc comparisons; ${ }^{*}, \mathrm{p}<0.05 ;{ }^{* \star}, \mathrm{p}<0.01 ;{ }^{* \star *}, \mathrm{p}<0.001, \mathrm{~ns}$, not significant. (g) Overview of lumenal domain construct purified from

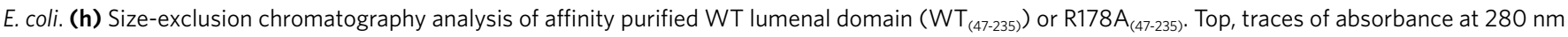
in mAu of WT and R178A lumenal domains. Bottom, SDS-PAGE analysis of 1-ml fractions by Coomassie staining. (i) Negative stain-EM analysis of WT lumenal domain oligomers shown in h. Right side shows 2D class averages. Size-bar, $500 \AA$. 
a

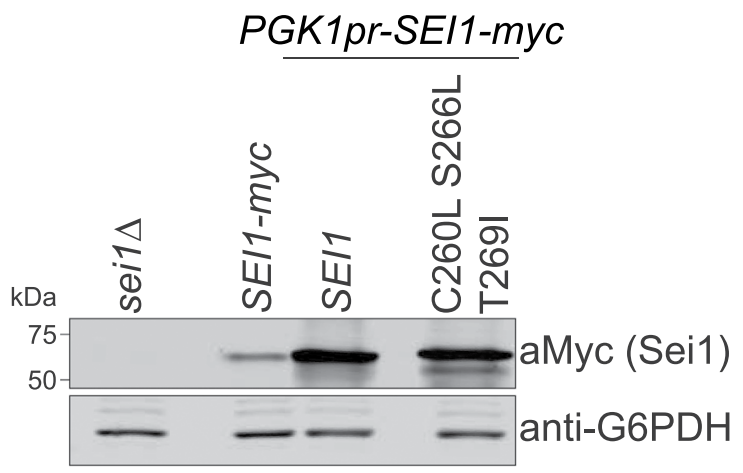

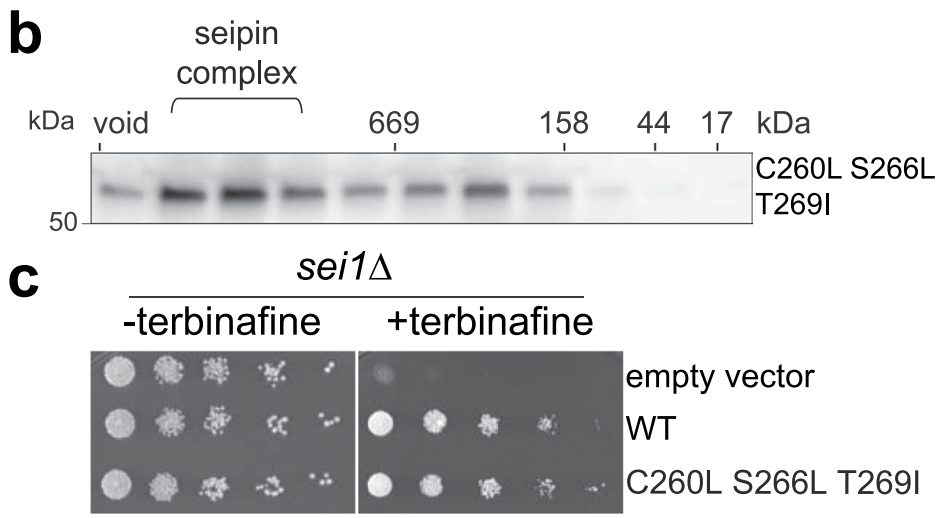

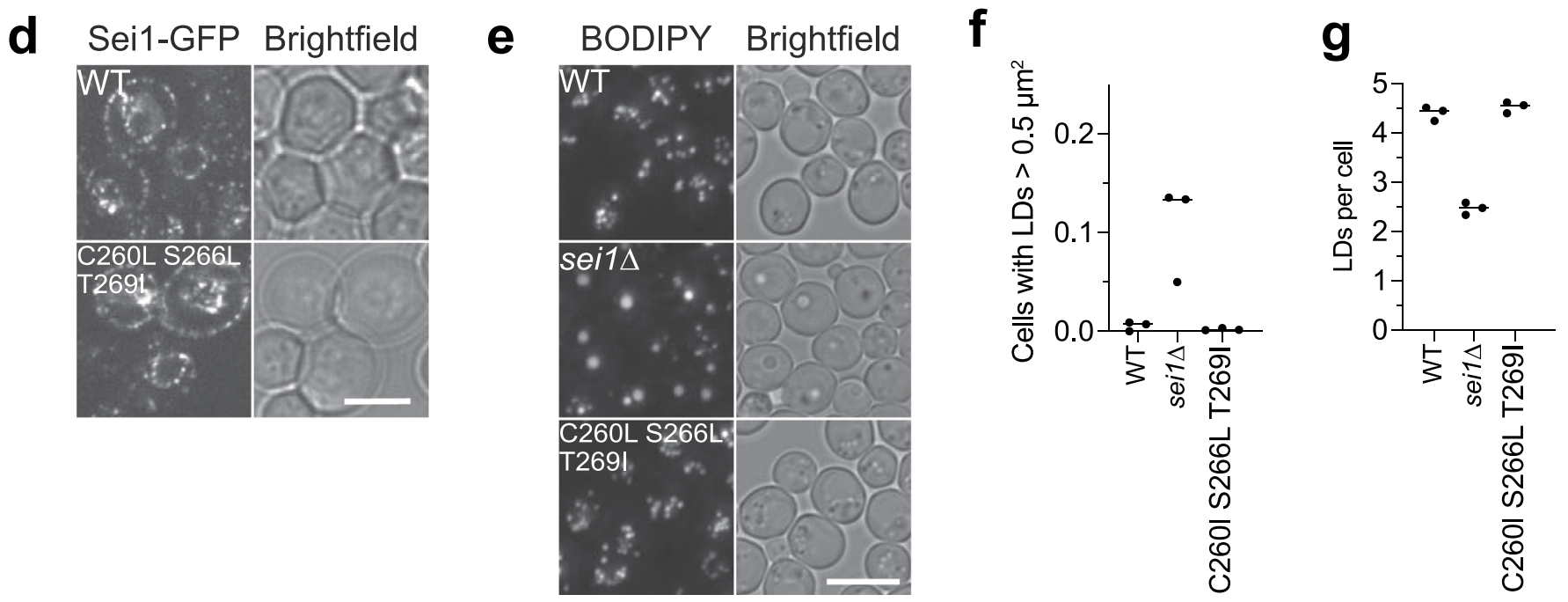

Extended Data Fig. 6 | Potential TG binding mutant retains function in vivo. (a) Western blot analysis of whole-cell lysates from strains expressing C-terminal 13xmyc tagged seipin from endogenous or PGK1 promoter. (b) Western blot analysis of fractions from size-exclusion chromatography of Triton X-100 solubilized membrane extracts carrying potential TG binding mutant C260L S266L T269I with C-terminal 13xmyc. (c) Growth of yeast strain sei14 carrying plasmids with C-terminally GFP-tagged SEl1 from yeast (WT), or indicated mutant on synthetic medium $\pm 100 \mu \mathrm{g} / \mathrm{ml}$ terbinafine. (d) Localization of seipin WT-GFP and C260L S266L T269I-GFP mutant expressed from plasmids in sei14 cells. Size bar, $5 \mu$ m (e) Analysis of LD morphology using BODIPY staining. Seipin mutants with C-terminal 13xmyc tag were expressed from PGK1 promoter. Size bar $=5 \mu \mathrm{m}$. (f,g) Quantification of experiment in panel $d . n=3$ biologically independent experiments. 

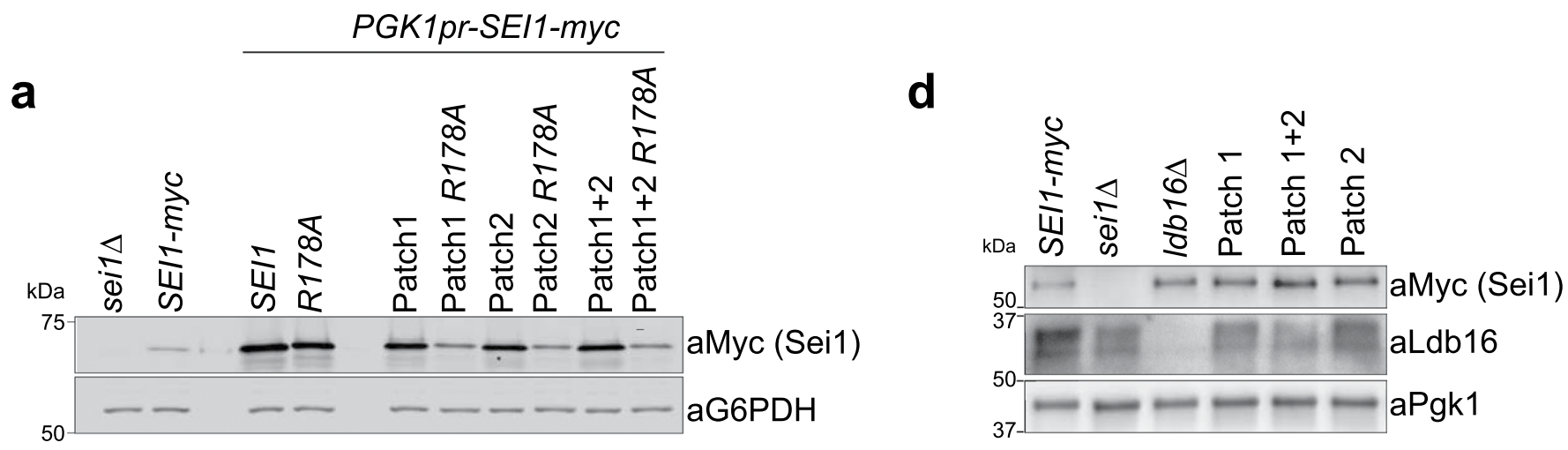

b

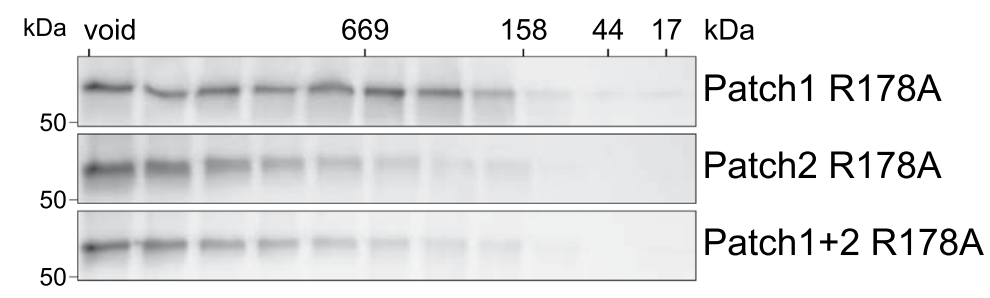

C

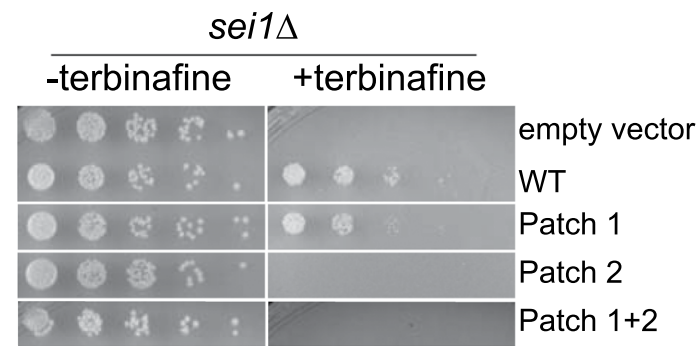

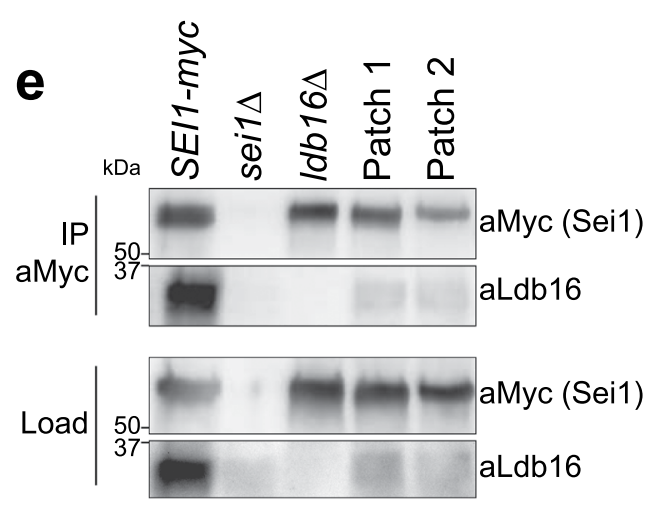

Extended Data Fig. 7 | Intramolecular transmembrane segment interactions are crucial for seipin function. (a) Western blot analysis of whole-cell lysates from strains expressing C-terminal 13xmyc tagged seipin variants from endogenous or PGK1 promoter. (b) Western blot analysis of fractions from size-exclusion chromatography of Triton X-100 solubilized membrane extracts carrying Patch mutants combined with R178A and C-terminal 13xmyc. (c) Growth of yeast strain sei1 $\Delta$ carrying plasmids with C-terminally GFP-tagged SEI1 from yeast (WT), or indicated mutants. (d) Western blot analysis of whole-cell lysates from strains expressing indicated seipin mutants under control of the PGK1 promoter and C-terminal 13xmyc tag. Representative immunoblots of two biologically independent experiment repeats is shown. (e) Immuno-precipitation of indicated seipin mutants via anti-myc resin. Equal amounts of load (detergent solubilized membranes in Tx100) and eluate fractions were loaded. $n=2$ biologically independent repeats. 
a

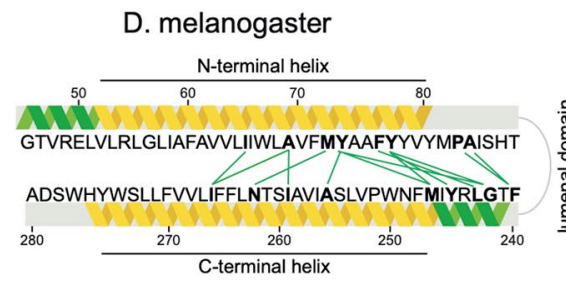

b

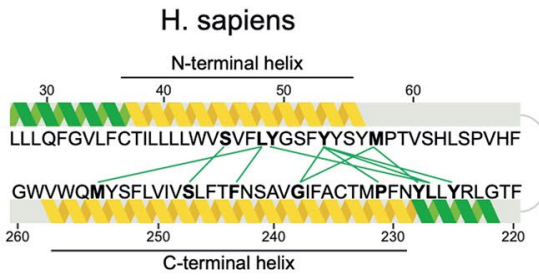

C

\begin{tabular}{|c|c|c|c|c|c|}
\hline \multicolumn{6}{|c|}{ sei1 } \\
\hline - terbinafine & & terb & in & & \\
\hline 00 端 & 6 & & & & \\
\hline 00 放: . & 0 & -0 & * & $\%$ & \\
\hline 00 和 & - & 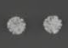 & * & $\%$ & \\
\hline $00+4$ & & 0 & $x$ & 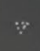 & \\
\hline $00 \%$ : & & - & 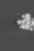 & 3 & \\
\hline 00 然 & & - 4 & 8 & $\because$ & \\
\hline 0.0 和 & & - & $?$ & & \\
\hline 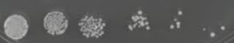 & & - & s & $\theta^{*}$ & \\
\hline
\end{tabular}

empty vector

WT

dmSeipin

yeast lumenal-dm TMD

dm lumenal-yeast TMD

hSeipin

yeast lumenal-human TMD

human lumenal-yeast TMD

d
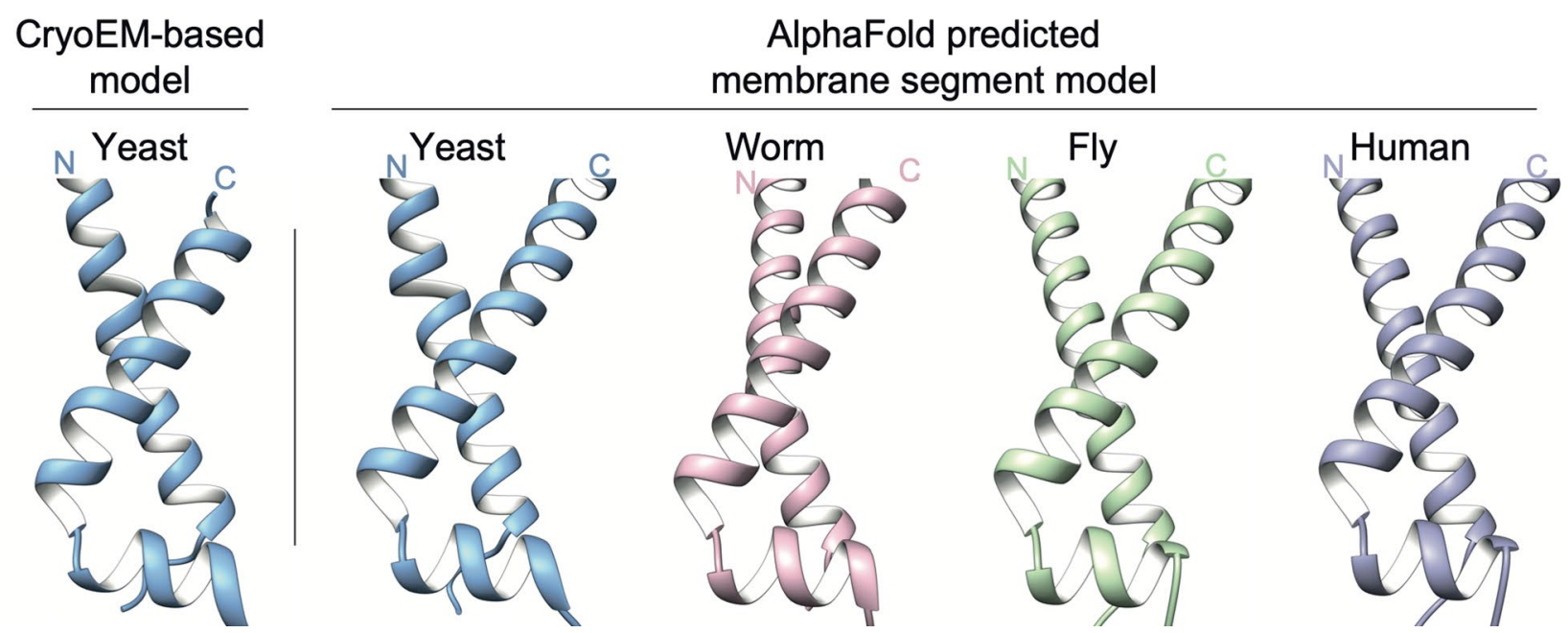

Extended Data Fig. 8 | Transmembrane segment architecture is conserved. (a,b) Highest ranking evolutionary couplings (green lines) within seipin transmembrane and switch regions mapped onto (a) D. melanogaster or (b) human sequences. Yellow and green helices indicate secondary structure prediction by Phyre $2^{57}$ of membrane embedded or hydrophilic helices, respectively. Coupling residues are indicated in bold. (c) Growth of yeast strain sei1 $\Delta$ carrying plasmids with C-terminally GFP-tagged SEl1 from yeast (WT), D. melanogaster (dmSeipin), human (hSeipin) or chimeric constructs on synthetic medium $\pm 100 \mu \mathrm{g} / \mathrm{ml}$ terbinafine. (d) The architecture of seipin transmembrane helices is predicted to be conserved. Comparison of switch and transmembrane regions of our structural model (left) with predicted structure of yeast (S. cerevisiae); worm (C. elegans), fly (D. melanogaster) or human by AlphaFold ${ }^{58}$. 
a

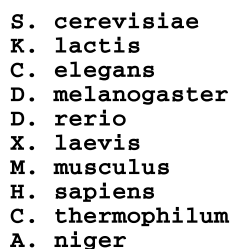

S. cerevisiae

Delanogaster

D. rerio

H. sapiens

A. niger b

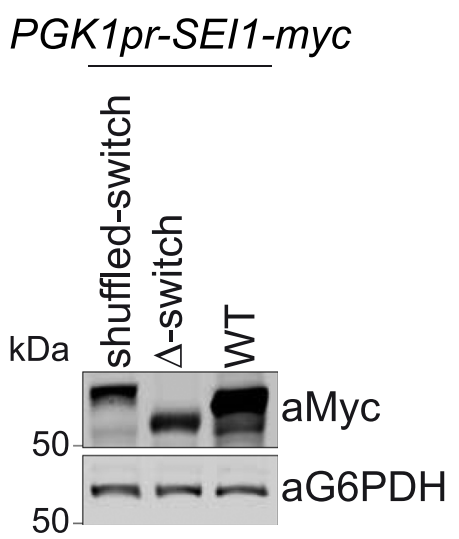

Extended Data Fig. 9 | Switch regions are required for seipin function. (a) Sequence alignment of seipin sequences from different species shows conserved $\mathrm{F}_{232} \times x G L R$ sequence motif. Identical residues are colored in red boxes, red characters and blue framed residues indicate similarity in a group or across groups, respectively. (b) Western blot analysis of whole cell lysates from strains expressing indicated switch mutants or WT seipin under control of the PGK1 promoter with C-terminal 13xmyc tag. 


\section{Reporting Summary}

Nature Portfolio wishes to improve the reproducibility of the work that we publish. This form provides structure for consistency and transparency in reporting. For further information on Nature Portfolio policies, see our Editorial Policies and the Editorial Policy Checklist.

\section{Statistics}

For all statistical analyses, confirm that the following items are present in the figure legend, table legend, main text, or Methods section.

$\mathrm{n} / \mathrm{a} \mid$ Confirmed

$\bigotimes$ The exact sample size $(n)$ for each experimental group/condition, given as a discrete number and unit of measurement

$\square$ A statement on whether measurements were taken from distinct samples or whether the same sample was measured repeatedly

The statistical test(s) used AND whether they are one- or two-sided

Only common tests should be described solely by name; describe more complex techniques in the Methods section.

Х $\square$ A description of all covariates tested

Х $\square$ A description of any assumptions or corrections, such as tests of normality and adjustment for multiple comparisons

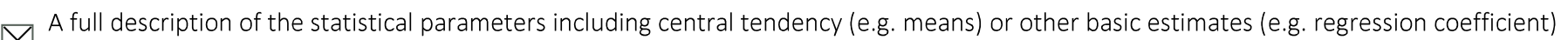
AND variation (e.g. standard deviation) or associated estimates of uncertainty (e.g. confidence intervals)

For null hypothesis testing, the test statistic (e.g. $F, t, r$ ) with confidence intervals, effect sizes, degrees of freedom and $P$ value noted Give $P$ values as exact values whenever suitable.

Х $\square$ For Bayesian analysis, information on the choice of priors and Markov chain Monte Carlo settings

Х $\square$ For hierarchical and complex designs, identification of the appropriate level for tests and full reporting of outcomes

$\bigotimes \square$ Estimates of effect sizes (e.g. Cohen's $d$, Pearson's $r$ ), indicating how they were calculated

\section{Our web collection on statistics for biologists contains articles on many of the points above.}

\section{Software and code}

Policy information about availability of computer code

Data collection SerialEM version 3.6; NIS-Elements AR (v4.51.01)

Data analysis Phenix (v 1.18); Coot (v 0.95); MotionCor2 (v 1.1.0); CTFFIND4 (v 4.1.5); SAMUEL (v 17.05); SamViewer (v 16.01); SPIDER (v 17.05); RELION 3.0; Resmap (v 1.1.4); bfactor (v 1.03); Chimera (v 1.13); ImageJ (v 2.0); GraphPad PRISM (v 9); Image Studio (v 5.2.5); custom code to segment cells is made publicly available at: $h$ ttps://github.com/hci-unihd/YeastCellSeg

For manuscripts utilizing custom algorithms or software that are central to the research but not yet described in published literature, software must be made available to editors and

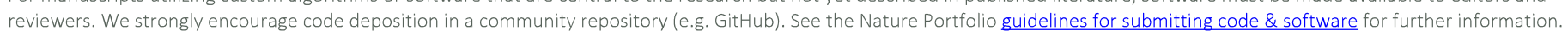

\section{Data}

Policy information about availability of data

All manuscripts must include a data availability statement. This statement should provide the following information, where applicable:

- Accession codes, unique identifiers, or web links for publicly available datasets

- A description of any restrictions on data availability

- For clinical datasets or third party data, please ensure that the statement adheres to our policy 
Please select the one below that is the best fit for your research. If you are not sure, read the appropriate sections before making your selection.

\ Life sciences

Behavioural \& social sciences

Ecological, evolutionary \& environmental sciences

For a reference copy of the document with all sections, see nature.com/documents/nr-reporting-summary-flat.pdf

\section{Life sciences study design}

All studies must disclose on these points even when the disclosure is negative.

Sample size No statistical methods were used to predetermine sample size. Sample sizes were chosen based on similar studies in the field.

Data exclusions No data were excluded from analyses.

Replication Each experiment was repeated at least two times in independent experiments. All attempts at replication were successful.

Randomization Not applicable to our study, as there was no assignment to different groups.

Blinding Not applicable to our study, as there was no assignment into groups.

\section{Reporting for specific materials, systems and methods}

We require information from authors about some types of materials, experimental systems and methods used in many studies. Here, indicate whether each material, system or method listed is relevant to your study. If you are not sure if a list item applies to your research, read the appropriate section before selecting a response.

\begin{tabular}{l|l} 
Materials \& experimental systems \\
\hline $\mathrm{n} / \mathrm{a}$ & Involved in the study \\
\hline & $\bigotimes$ Antibodies \\
$\square$ & $\square$ Eukaryotic cell lines \\
$\square$ & $\square$ Animals and other organisms \\
$\square$ & $\square$ Human research participants \\
$\square$ & $\square$ Clinical data \\
$\square$ & $\square$ Dual use research of concern
\end{tabular}

Methods

$\mathrm{n} / \mathrm{a}$ Involved in the study

Х $\square$ ChIP-seq

Х $\square$ Flow cytometry

Х $\square$ MRI-based neuroimaging

\section{Antibodies}

Antibodies used

anti-myc monoclonal 9E10 (Cat\# MA1-980; Thermofisher); anti-G6PDH (Cat\#A-9521; Sigma); goat anti-mouse-IRDye (Cat\# 926-32210; Licor); goat anti-rabbit-IRDye (Cat\# 926-32211, Licor); m-IgGk BP-HRP (Cat\# sc-516102; Santa Cruz Biotechnology); mouse anti-rabbit IgG-HRP (Cat\# sc-2357; Santa Cruz Biotechnology); Mouse monoclonal anti-Pgk1 (Cat\# 459250; Invitrogen); Mouse monoclonal anti-FLAG (Cat\# F1804; Sigma); anti-Ldb16 rabbit polyclonal (Wang et al., 2014), gift from Chao-Wen Wang (Institute of Plant and Microbial Biology, Academia Sinica, Taipei City).

Validation

anti-Ldb16 antibodies were verified on western blots of cell lysate from WT vs. Idb16 $\Delta$ cells and were previously validated in Wang et al., 2014. Validations of commercially available antibodies can be found at the manufacturers sites: anti-myc monoclonal (https:// www.thermofisher.com/antibody/product/c-Myc-Antibody-clone-9E10-Monoclonal/MA1-980); anti-G6PDH (https:// www.sigmaaldrich.com/US/en/product/sigma/a9521); anti-Pgk1 (https://www.thermofisher.com/antibody/product/PGK1-Antibodyclone-22C5D8-Monoclonal/459250); anti-FLAG (https://www.sigmaaldrich.com/US/en/product/sigma/f3165). 